\title{
Modulation of the $\mathrm{IK}_{\mathrm{S}}$ channel by $\mathrm{PIP}_{2}$ requires two binding sites per monomer
}

Audrey Deyawe Kongmeneck ${ }^{1}$, Marina A. Kasimova ${ }^{1}$, and Mounir Tarek ${ }^{1 *}$

1 Université de Lorraine, CNRS, LPCT, F-54000 Nancy, France

*Correspondence: mounir.tarek@univ-lorraine.fr 


\section{Abstract}

The phosphatidyl-inositol-4,5-bisphosphate $\left(\mathrm{PIP}_{2}\right)$ lipid has been shown to be crucial for the coupling between the voltage sensor and the pore of the potassium voltage-gated $\mathrm{Kv} 7$ channel family, especially the Kv7.1 channel. The latter, expressed in the myocardium membrane is complexed with its auxiliary subunits, KCNE1 to generate the so-called IKs current. We present here molecular models of transmembrane domain of this complex in its three known states, namely the Resting/Closed (RC), the Intermediate/Closed (IC), and the Activated/Open $(\mathrm{AO})$, robustness of which is assessed by agreement with a range of biophysical data. Molecular Dynamics (MD) simulations of these models embedded in a lipid bilayer including phosphatidyl-inositol-4,5-bisphosphate $\left(\mathrm{PIP}_{2}\right)$ lipids show that in presence of KCNE1, two PIP2 lipids are necessary to stabilize each state. The simulations also show that $\mathrm{KCNE} 1$ interacts with both $\mathrm{PIP}_{2}$ binding sites, forming a tourniquet around the pore and preventing its opening. The present investigation provides therefore key molecular elements that govern the role of $\mathrm{PIP}_{2}$ in KCNE1 modulation of $\mathrm{IK}_{s}$ channels, possibly a common mechanism by which auxiliary KCNE subunits might modulate a variety of other ion channels. 


\section{Introduction}

The IKs current is diffused through the plasma membranes of cardiomyocytes, during the last (fourth) phase of the cardiac action potential (Barhanin et al., 1996; Nerbonne and Kass, 2005; Tristani-Firouzi and Sanguinetti, 1998). This repolarization current is conducted by a tetrameric protein complex derived from the co-expression of four $\mathrm{Kv7.1}$ a-subunits and KCNE1 ancillary subunits respectively from the KCNQ1 and KCNE1 genes. The Kv7.1 tetramer forms a voltage-gated potassium (Kv) channel, a transmembrane protein that upon modification of membrane potential, opens and carries selectively potassium ions to the extracellular medium, while KCNE1 is a transmembrane peptide which acts as an ancillary $\beta$-subunit for several Kv channels besides the Kv7.1 one (McCrossan and Abbott, 2004). Together, the $\alpha$ and $\beta$ subunits are forming the IKs channel complex. Kv7.1 channels are homo-tetramers of 6 transmembrane helical segments, the first four ones (S1 to S4) forming the voltage-sensor domain (VSD), and the last two (S5 and S6) forming the pore domain (PD) of the channel. As for most Kv channels, the VSD and PD are swapped in Kv7.1. The segment S4 in the VSD carries four positively charged arginines called gating charges that move across the membrane upon depolarization, interacting sequentially with negatively charged side chains from S2 (Wu et al., 2010a). The Kv7.1 subunit also contains a cytoplasmic CTERM region composed of four cytosolic helices, the first two being connected to the sixth transmembrane segment, the last two being located deeper in the cytosol, forming the tetramerization domain (Wiener et al., 2008). KCNE1 on the other hand is a transmembrane polypeptide of 129 aminoacids divided into one extracellular NTERM domain, a helical transmembrane (TMD) domain, and a cytosolic CTERM domain (Tian et al., 2007). 
Numerous mutations of both KCNQ1 and KCNE1 genes are associated with long QT syndromes (LQTS) (Kapplinger et al., 2009; Napolitano et al., 2005; Splawski et al., 2000). The latter are characterized by an extended cardiac action potential that corresponds to the time interval between $Q$ and T waves in electrocardiograms. The LQT phenomenon reflects the inability of the protein complex to generate its IKs outward current and therefore to return cardiomyocytes membranes toward their resting potential. This delay in repolarization disturbs the propagation of the cardiac action potential within the myocardium tissue, and therefore leads to heart rhythm abnormalities, also known as cardiac arrhythmias. Hence, the IKs channel is a therapeutic target for the treatment of LQTS, whose function must be studied and understood to be able to develop any potential effective drug. Over the last twenty years, this protein complex has been extensively studied using various methods (Liin et al., 2015).

The gating of Kv7.1 and IKs channels is triggered by membrane depolarization and involves three stable states of the VSD: Resting, Intermediate, and Activated. The conformations of these states are known to transition from one to another through the motion of three S4 gating charges, R228 (R1), R231 (R2) and R237 (R4) with respect to two binding sites (Table S1). As in VSDs of most voltage-gated ion channels, that of Kv7.1 contains two strongly conserved binding sites (residues forming salt bridges with the S4 gating charges): the first one, located in the solvent accessible surface of the VSD, is an acidic residue from S2, E160 (E1). The second one, located deeper in the membrane, is sheltered from the solvent by an aromatic residue from S2, F167, also known as the charge transfer center (CTC), and is composed of two acidic residues, E170 (E2) and D202 (D3) from S2 and S3 segments, respectively (Tao et al., 2010). 
These conformational changes also involve a translation of these gating charges through an aromatic residue from S2, F167, which was suggested to constitute the interface between the solvent accessible surface of the VSD, and its occluded site. This residue is conserved in homologous Kv channels such as Kv1.2 or Kv2.1 (Lacroix and Bezanilla, 2011).

The pore opening is elicited by another mechanism called VSD-PD coupling. The latter occurs in most Kv channels and is governed by protein-protein interactions between VSD and PD of distinct $\alpha$-subunits that couple the activation state of the VSD to the conformation (open or closed) of the PD (Roux, 2006). The exact mechanism for this "electromechanical" process is not completely determined, yet a functional study revealed that the VSD-PD coupling mechanism of the Kv7.1 tetramer occurs in both the Activated and the Intermediate states of the VSD, but not in its Resting state (Hou et al., 2017). In our recent integrative study, we unveiled the molecular determinants of the distinct intersubunit coupling interfaces that underlie the VSD-PD coupling in the Intermediate and the Activated states of the Kv7.1 channel (Hou et al., 2020). In presence of KCNE1, this mechanism appears to be hindered when the VSD is in its Intermediate state and enhanced in its Activated state. Hence, the three functional states known for IKs channel are described by the "activation state" of the $\operatorname{VSD}(\mathrm{R}, \mathrm{I}$ or $\mathrm{A})$ and by the conformation $(\mathrm{O}$ or $\mathrm{C})$ of the pore, and are referred to as $\mathrm{RC}, \mathrm{IC}$ and $\mathrm{AO}$ states. 
The phosphatidyl-inositol-4,5-bisphosphate $\left(\mathrm{PIP}_{2}\right)$ is a membrane phospholipid which participates in the function of many membrane transporters (Hilgemann et al., 2001), including voltage-gated ion channels. $\mathrm{PIP}_{2}$ is present at a $1 \%$ rate in the inner leaflet of the lipid bilayers forming cell membranes, and experimental studies have shown that it interacts with numerous ion channels including $\mathrm{K}_{v}$ channels to modulate their activation. This lipid has been shown to be crucial for the coupling between the VSD and the PD for the Kv7 channel family (Li et al., 2005; Zaydman and Cui, 2014), especially in the Kv7.1 channel (Zaydman et al., 2013, 2014).

Hence for instance, the structure of Xenopus Laevis Kv7.1 monomer (KCNQ1EM), resolved by cryo-electron microscopy (CryoEM), was locked in a non-physiological conformation of the PD, namely the uncoupled Activated/Closed (AC) state (Sun and MacKinnon, 2017). According to the authors of the study, this might be explained by the absence of $\mathrm{PIP}_{2}, \mathrm{known}$ to be essential for the coupling of the VSD activated state with PD open state in the Kv7 channels family (Li et al., 2005). 
Since beside KCNQ1EM (Sun and MacKinnon, 2017), and the very recently published CryoEM structures of the human Kv7.1 AO channel in the presence of $\mathrm{PIP}_{2}$ (Sun and MacKinnon, 2020) , no high-resolution structure of the IKs channel is available, we and others turn to molecular modeling. Quite surprisingly, among the molecular constructs of the IKs channel that have been published the last decade (Gofman et al., 2012; Kang et al., 2008; Ramasubramanian and Rudy, 2018a; Xu et al., 2013), very few were modeled in presence of $\mathrm{PIP}_{2}$. Moreover, those which were modeled with the lipid aimed at validating experiments were limited to the identification of the PIP2 binding sites (Eckey et al., 2014), without providing any molecular insight about the way the lipid interacts with Kv7.1 or with its KCNE1 subunits. Recently, a homology model (Jalily Hasani et al., 2017) of the IKs complex was subjected to Molecular Dynamics (MD) simulations in a POPC:PIP 2 membrane at a 10:1 ratio (Jalily Hasani et al., 2018). Unfortunately, the $\mathrm{IK}_{s}-\mathrm{PIP}_{2}$ interactions were not extensively investigated and the study did not provide information about how $\mathrm{PIP}_{2}$ affects the VSD-PD coupling mechanism of the channel. Moreover, despite the fact that several studies have shown that IKs complexes can be expressed in cardiomyocytes with a 4:4 (Kv7.1:KCNE1) stoichiometry (Murray et al., 2016; Nakajo et al., 2010) most of the IKs models reported so far (Jalily Hasani et al., 2017; Kang et al., 2008; Xu et al., 2013) have not been built with this ratio. 
Our previous MD study (Kasimova et al., 2015) of Kv7.1 models in open and closed states, allowed one to localize the $\mathrm{PIP}_{2}$ binding site in the $\mathrm{Kv} 7.1$ subunit, and to characterize the key elements of the Kv7.1 modulation by $\mathrm{PIP}_{2}$ in absence of KCNE1. The lipid was shown to participate in VSD/PD coupling of the Kv7.1 channel through state-dependent interactions, preventing repulsive forces between basic residues from the S2-S3LOOP and S4 in resting state, and between basic residues from the S2-S3Loop and S6 in open states. These MD simulations also suggested that $\mathrm{PIP}_{2}$ may constitute a third binding site for $\mathrm{S} 4$ gating charges. Indeed, the lipid was found to form salt-bridges with R237 (R4) and R243 (R6) in the RC model of Kv7.1, and not in the AO model. Moreover, the dependence of this lipid for the function of Kv7.1 was also proved to be increased in presence of KCNE1 due to its additional positive charges in its CTERM domain (Li et al., 2011). This study indicates that IKs may carry an additional $\mathrm{PIP}_{2}$ binding site with respect to $\mathrm{Kv} 7.1$. Therefore, the molecular determinants describing a second $\mathrm{PIP}_{2}$ binding site in $\mathrm{Kv} 7.1$ subunits in presence of KCNE1 are yet to be investigated. To address these questions, computational chemistry methods are the most insightful ones to unravel the elements of protein function at a molecular level of precision.

Here we propose three models of the Kv7.1 tetramer in distinct metastable states, in presence of KCNE1 subunits corresponding to the RC, IC and AO states of the IKs channel. Each model has been studied either with one or two $\mathrm{PIP}_{2}$ lipids per Kv7.1 subunit. A total of 6 systems (Figure 1) were submitted each to $\sim 500 \mathrm{~ns} \mathrm{MD} \mathrm{simulations} \mathrm{to} \mathrm{fully} \mathrm{relax} \mathrm{the} \mathrm{initial}$ constructs. The analyses of these trajectories were then used to assess the validity of the models by comparing them to the literature. 


\section{Results}

Validation of the $\mathrm{IK}_{\mathrm{s}}$ models: $2 \mathrm{PIP}_{2}$ lipids per subunit are in better agreement with experiments

Molecular modeling methods are based upon the study of representations of chemical and biochemical entities. The main goal of using these computational methods, in the frame of this study, is to gain an atomistic insight on phenomena that were unraveled by experimental studies. Hence, to predict the molecular mechanisms associated to these phenomena, we need to maximize as much as possible the compliance of our models with respect to structural biology data. To achieve this goal, we built our 3D models using structural constraints that were directly drawn from experimental data, and then we monitored the stability of these constraints over the collection of conformations generated by MD simulations, i.e. the trajectories.

To gain a finer insight in the molecular determinants allowing for the stabilization of each state of Kv7.1 and IKs channels, MD simulations were performed to equilibrate each molecular model within POPC membrane and $\mathrm{PIP}_{2}$ lipids. In various $\mathrm{Kv}$ channels, the activation mechanism of the VSD is mostly characterized by state-dependent salt-bridges between basic residues of S4 and acidic residues spread in S2 and S3 segments (Figure S1). This succession of salt-bridges is describing an upward translation of $S 4$, as well as a clockwise rotation during VSD activation. To validate the state dependent structures of our models VSDs, we monitored the distance between each pair of charge groups that are supposed to interact according to the literature. The state-dependent salt-bridges found in the VSD of our IKs models are reported in Table S1. 
In $8 \mathrm{PIP}_{2}$ systems, the interactions between $\mathrm{E} 1$ and R228 (R1), and between E2 and R231 $(\mathrm{R} 2)$, which are specific of the $\mathrm{RC}$ state, are both present specifically in our $\mathrm{RC}$ model in all subunits. In $\mathrm{RC}$ model with $4 \mathrm{PIP}_{2}$ system, the interaction between $\mathrm{R} 1$ and $\mathrm{E} 1$ is present, but the interaction between R2 and E2 is absent, as R2 interacts with E1 in only two subunits out of four. This first result indicates that the presence of two $\mathrm{PIP}_{2}$ binding sites in $\mathrm{IK}_{s}$ models may be crucial for the stabilization of the VSDs.

In the IC state, S4 translates upwards and rotate clockwise, which leads to E1 interacting with R231 (R2), while E2 interacts with R237 (R4). These interactions have been specifically found in IC model of 8PIP 2 system, in all subunits for E1-R2 interaction, and in 3 subunits out of 4 for $\mathrm{E} 2-\mathrm{R} 4$ interaction. In the $4 \mathrm{PIP}_{2}$ system, $\mathrm{E} 1-\mathrm{R} 2$ interaction is present as expected, but E2-R4 interaction is not absent. Interestingly, R4 also interacts with D3 in all subunits of both systems. The latter forms the charge transfer center along with F167 from S2 and E2. Therefore, R4-D3 interaction may indicate that R4 is fully anchored in the CTC in the 8PIP2 system and remain partially anchored in the CTC in the 4PIP 2 system.

In the $\mathrm{AO}$ state, a second upward translation and a clockwise rotation of S4 lead to E1-R4 interaction. This interaction is present only in our $\mathrm{AO}$ models of both $\mathrm{PIP}_{2}$ systems. In these models, R6 appeared to be anchored in the CTC in all subunits, interacting with both E2 and D3 (data not shown). Noteworthy, the pairs of salt-bridges determined in our IKs models are also present in their respective states of $\mathrm{Kv} 7.1$ models. These MD results highlight the different conformations of the VSD correspond to interaction patterns which remain very stable over time. However, in the $4 \mathrm{PIP}_{2}$ systems, state dependent salt-bridge pairs are satisfying experimental data for $\mathrm{AO}$ model, but not for IC and $\mathrm{RC}$ models. 
Overall, these results suggest that IKs models of $8 \mathrm{PIP}_{2}$ system are in better agreement with experimental results, as their state dependent expected VSD interactions are more satisfied, and more stable throughout the corresponding MD trajectories. Note that the distinct patterns of gating charge salt-bridges determined in our molecular models support the sliding helix VSD activation mechanism proposed for the Kv channels (Durell and Guy, 1992), as well as for the voltage-gated sodium channels (Catterall, 1986).

Besides electrostatic interactions, site-directed mutagenesis can provide important information about the relative position of the KCNE1 helices with respect to the $\mathrm{Kv} 7.1$ subunits. Experiments such as cysteine cross-linking mutagenesis allow one to spot the residues which are close enough to form disulfide bonds. In the framework of such experiments, a disulfide bond can be formed if the $\mathrm{C}_{\beta}$ atoms of the corresponding amino acids are within $13 \AA$ (Careaga and Falke, 1992). Thanks to such experimental studies, a significant amount of neighbor residue pairs within the Kv7.1 channel and the IKs complex have been reported. Accordingly, for each neighbor residue pair, we computed the distance between their respective $C_{\beta}$ atoms over our MD trajectories and assumed neighbor residue pairs present in the model if the latter was below $13 \AA$. Among the seven neighbor residue pairs from Kv7.1 (Table S2), the intersubunit pairs assigned to IC state, as well as the intrasubunit ones assigned to the RC state are present in all IKs models (Figure S2, A), regardless of their respective state and regardless of the amount of $\mathrm{PIP}_{2}$ lipids. For the three pairs assigned to the $\mathrm{AO}$ state (Figure S2, B), one of those is present in all models, whereas the other two are predominant in most subunits of the $\mathrm{AO}$ and $\mathrm{RC}$ models, but absent in the IC models. In our Kv7.1 models, all these pairs are also present in all subunits. 
For pairs involving cross linking between Kv7.1 and KCNE1 residues (Table S3), most of those which have been assigned to the $\mathrm{AO}$ state of $\mathrm{IK}$ channel are present in both $4 \mathrm{PIP}_{2}$ and $8 \mathrm{PIP}_{2} \mathrm{AO}$ models (Figure S3). Surprisingly, the only pair of residues assigned to $\mathrm{AO}$ state and not present in our AO model (Gln147-Lys41) is present in the RC models only. Among the six residue pairs assigned to the $\mathrm{RC}$ state, five of those are present in the $8 \mathrm{PIP}$ system, against four of them in the 4PIP 2 one. The pairs of residues which are not interacting in $\mathrm{RC}$ models are not present in any other model. Finally, for the nine remaining pairs, which were not assigned to any state of the channel, all of them are present in at least one model of each system. Among the three residue pairs that include Kv7.1 S6 residues and KCNE1 Cterm residues, only one pair is present in both systems. The second pair is present in the 4PIP 2 system only, and the last pair is absent in both systems.

Overall, Kv7.1-Kv7.1 and Kv7.1-KCNE1 residue pairs are mostly present in all IKs models, regardless of the number of $\mathrm{PIP}_{2}$ lipids in the system. Although the presence and the stability of the interactions between each residue pair in our models is testifying for their robustness, these results alone cannot allow to select the best IKs models among their distinct systems.

- Neighbor residue pairs: protein-lipid interactions

Besides the protein-protein interactions, several mutagenesis studies conducted on Kv7.1 in absence and presence of KCNE1 also have highlighted ten basic residues from Kv7.1 and three others from KCNE1 which can possibly interact with $\mathrm{PIP}_{2}$ (Table S4). To characterize these interactions in our IKs models, we calculated average distances between charged groups of each basic residue and $\mathrm{PIP}_{2}$. Among the thirteen $\mathrm{Kv} 7.1$ and $\mathrm{KCNE} 1$ basic residues which have been shown to be involved in electrostatic interactions with the lipid, ten residues interact with $\mathrm{PIP}_{2}$ in at least one MD trajectory of $\mathrm{IK}_{s}$ model in $8 \mathrm{PIP}_{2}$ system. 
Results obtained for these models showed that $\mathrm{PIP}_{2}$ inter and $\mathrm{PIP}_{2}$ intra are specifically anchored to the VSD and the PD, respectively, in a state independent manner (Figure S4). In each model, residues R190 from the S2-S3LOOP and R249 from the S4-S5LINKER interact with $\mathrm{PIP}_{2}$ intra, while residues $\mathrm{K} 362$ and $\mathrm{R} 366$ from S6 interact with $\mathrm{PIP}_{2}$ inter. In addition, some basic residues are also interacting with $\mathrm{PIP}_{2}$ in a state dependent manner, such as R192 and R195 from S2-S3Loop, as well as K358 from S6, whose interactions with PIP 2 are favored in AO model, as well as in IC model for R195. State independent interactions involving VSD residues are related to a motion of $\mathrm{PIP}_{2}$, which appears to progressively anchor S2-S3LOOP, while remaining bound to S4-S5LINKER residue R249 during VSD activation. State dependent interactions involving PD residues are related to the movement of S6 upon pore opening. $\mathrm{PIP}_{2}$ inter progressively binds basic residues of $\mathrm{S} 6$ cytoplasmic helices as they spread away from the pore axis towards inner membrane surface where $\mathrm{PIP}_{2}$ is localized. Oppositely, the interaction between residue R243 from S4 and the lipid is favored in RC model, which suggests that R243 loses its interaction with $\mathrm{PIP}_{2}$ as S4 translates upward during the VSD activation. In IKs models of the 4PIP 2 system, state-independent interactions between VSD residues and $\mathrm{PIP}_{2}$ intra are present, but those between $\mathrm{S} 6$ residues and $\mathrm{PIP}_{2}$ are merely present. Contrary to Kv7.1 models in which $\mathrm{PIP}_{2}$ intra can bind $\mathrm{S} 6$ in $\mathrm{AO}$ model, K354 is the only S6 residue that is able to bind $\mathrm{PIP}_{2}$ in both $\mathrm{IC}$ and $\mathrm{AO}$ models of IKs complex. Residues K358, K362 and R366 are located too far away to reach $\mathrm{PIP}_{2}$ intra in any of these models.

Four residues are not interacting with $\mathrm{PIP}_{2}$ in any $\mathrm{IK}$ s system. Residues $\mathrm{R} 181$ and K196 from S2-S3 sidechains remain tangent to the conduction pathway, was unable to interact with $\mathrm{PIP}_{2}$ inter. R259 guanidium group, despite being close to $\mathrm{PIP}_{2}$ lipids, cannot get close to the phosphoryl groups of $\mathrm{PIP}_{2}$ because of the steric hindrance induced by presence of KCNE1 subunits. 
Surprisingly, the KCNE1 residues R67, K69 and K70 have their sidechains oriented in two opposite directions, allowing for the ancillary subunit to interact with both $\mathrm{PIP}_{2}$ binding sites in a state-dependent fashion in IKs models of $8 \mathrm{PIP}_{2}$ system. In RC model, residue K69 binds $\mathrm{PIP}_{2}$ intra in two opposite subunits, while $\mathrm{R} 67$ and $\mathrm{K} 70$ are both binding $\mathrm{PIP}_{2}$ inter in all subunits. In IC model, the interaction with $\mathrm{K} 70$ is absent, while those with both R67 and K69 are still present. In AO model, K69 is the only KCNE1 residue interacting with $\mathrm{PIP}_{2}$. These subsequent interactions over all our stable state models of IKs of $8 \mathrm{PIP}_{2}$ system suggest that KCNE1 undergoes two clockwise rotations during VSD activation, each occurring during the $\mathrm{RC}-\mathrm{IC}$ and the IC-AO transitions, respectively. In the IC and RC models, KCNE1 residues are binding both inter and intra $\mathrm{PIP}_{2}$, forming a circle around the cytoplasmic region of S6 helices. This $\mathrm{PIP}_{2}-\mathrm{KCNE} 1$ circle was not observed in the $\mathrm{AO}$ model, as KCNE1 does not bind $\mathrm{PIP}_{2}$ intra. Thus, the cytoplasmic region of $\mathrm{S} 6$ can spread away from the pore axis towards $\mathrm{PIP}_{2}$ inter. Quite interestingly, in the MD trajectories of $\mathrm{IK}$ s models in the $4 \mathrm{PIP}_{2}$ system, a likewise $\mathrm{PIP}_{2}-\mathrm{KCNE} 1$ circle cannot be formed due to the absence of $\mathrm{PIP}_{2}$ inter. Indeed, interactions involving R67 and K70 are impaired, while those involving K69 are conserved. However, in the $\mathrm{AO}$ model, the interaction between $\mathrm{K} 69$ and $\mathrm{PIP}_{2}$ is impaired, and basic residues of KCNE1 are facing those of $\mathrm{S} 6$ that strongly interact with $\mathrm{PIP}_{2}$ inter in $8 \mathrm{PIP}_{2}$ systems, which might generate electrostatic repulsions, leading to the collapse of S6 segments and pore closure.

In summary, our results highlighted an additional $\mathrm{PIP}_{2}$ binding site in $\mathrm{IK}$ s channel, with which KCNE1 interacts in a state dependent manner. This additional $\mathrm{PIP}_{2}$ binding site allow for the shaping of a $\mathrm{PIP}_{2}-\mathrm{KCNE} 1$ circle in the uncoupled $\mathrm{RC}$ and IC states model of IKs channel. This may allow for the control of S6 helices conformations by the KCNE1 subunits. 
To verify this hypothesis, results of pore radii calculations in our IKs models turn out to be useful. Indeed, results obtained for the IKs models will allow to figure out if the presence of $\mathrm{PIP}_{2}$ inter in the $8 \mathrm{PIP}_{2}$ systems is inducing a change in the width of the conduction pathway.

\section{- Pore radii}

Pore radii calculations have been conducted to select the best IKs model among the systems with $4 \mathrm{PIP}_{2}$ lipids and the ones with $8 \mathrm{PIP}_{2}$ lipids. Based on the correlation identified between the pore size of a Kv channel and the free energy associated to the conduction of a potassium ion (Treptow and Tarek, 2006), the analysis of the pore size of our models can allow to assess the reliability of our IKs models with respect to those of $\mathrm{Kv} 7.1$ model, and also with respect to experimental data. Integrative studies conducted on the Shaker channel (Díaz-Franulic et al., 2015) highlighted the fact that the cytoplasmic region of Kv channel conduction pathway need to be able to accommodate a solvated potassium ion, whose minimal radius is $\sim 3.6 \AA$, to be open. Pore radius measurement of the conduction pathway of KCNQ1EM structure (Sun and MacKinnon, 2017) in its Activated/Closed state reveals three S6 regions of constriction in the cytoplasmic side of the conduction pathway: the upper constriction is formed by the backbones of G345; the middle constriction is formed by hydrogen bonds between S349 hydroxyl groups, and the lower constriction is formed by the L353 side chains that are oriented towards the center of the pore and binding each other to form a hydrophobic seal. These three regions of constriction present pore radii of $\sim 2 \AA$ for $\mathrm{G} 345, \sim 0.8 \AA$ for S349, and $\sim 1.15 \AA$ for $\mathrm{L} 353$, which may prevent a $\mathrm{K}^{+}$ion to go through this pathway, as its ionic radius is $\sim 1.33 \AA$. This pore radius profile provides a hint on the constriction zones of Kv7.1 pore when the channel is decoupled due to the absence of $\mathrm{PIP}_{2}$ lipids. Hence, we compared first the pore radii profiles of IKs MD trajectories in its distinct systems, and then we compared the most robust model of IKs in its three states with those of $\mathrm{Kv} 7.1$ models. 
We mapped the average pore radii to see if these residues are forming a constriction in our IKs models. The MD trajectories of our models suggest that the backbone of G345 is oriented toward the center of the pore, while the side chains of both S349 and L353 remain tangent to the pore surface in all models. Nevertheless, the average pore radii estimated on these regions (Figure2) shed light on state dependent opening / closure of the conduction pathways. The models indicate, in the $\mathrm{AO}$ models (Figure $2 \mathrm{~A}$ ), values are between $2.7 \AA$ and $5 \AA$. In the Intermediate models (Figure $2 \mathrm{~B}$ ), these values are between $2 \AA$ and $4.5 \AA$. In the resting models (Figure $2 \mathrm{C}$ ), the average pore radii at the level of constricted regions assume values between $1 \AA$ and $3 \AA$ for the three models.

To discriminate one IKs model from another, we compared for each state, the average pore radii values obtained at the level of the constriction zones formed by G345, S349 and L353 in IKs models with those of KCNQ1EM structure. In the $A O$ state, Kv7.1 ionic current is increased in presence of KCNE1, thus IKs AO models should present wider pore radii than KCNQ1EM structure at the level of constriction regions. Among our AO models (Figure 2A), the $\mathrm{IK}_{s} 8 \mathrm{PIP}_{2}$ system have the least constrictions. Pore radii values of $\sim 3 \AA$, $3.9 \AA$ and $4.5 \AA$ in average at the levels of G345, S349 and L353, were respectively found. The IKs 4PIP $2 \mathrm{AO}$ model in contrast appears to be more constricted at the level of S349, as its average pore radii is $0.9 \AA$ lower than those of other $\mathrm{AO}$ models. A decrease of $1 \AA$ in the radius value of a nanopore can lead to a significant rise in the free energy cost necessary for conduction (DíazFranulic et al., 2015; Treptow and Tarek, 2006). This result, in addition to the number of protein-lipid interactions we found in $\mathrm{IK} s \mathrm{AO}$ models, suggest that the $\mathrm{IK} s \mathrm{AO}$ model is in better agreement with experimental data when embedded in $8 \mathrm{PIP}_{2}$ system than in $4 \mathrm{PIP}_{2}$ system. 
In the Intermediate state, the ionic current is abolished in presence of KCNE1, so IKs models should present a smaller pore radius than in Kv7.1. In the intermediate models (Figure 2B), the pore radii of the constriction zone of $L 353$ are $\sim 3.6 \AA$ in all models. Therefore, we only considered G345 and S349 constriction zones. The Kv7.1 model present the highest average pore radii values, ( $2.7 \AA$ and $2.9 \AA$ at the levels of G345 and S349, respectively) compared to the IKs models (both $\sim 2 \AA$ ). Hence, in a nutshell, the results obtained for intermediate models suggest that Kv7.1 conduction pathway is $\sim 0.8 \AA$ narrower in presence of KCNE1, regardless of the number of $\mathrm{PIP}_{2}$ lipids in the system which agrees with experiments.

In summary, constriction zones of $\mathrm{RC}$ models (Figure $2 \mathrm{C}$ ), present pore radii values below the minimal hydrodynamic radius of $\mathrm{K}^{+}$ions of $3.6 \AA$, which suggest that in $\mathrm{RC}$ models, the pore is closed regardless of the presence of KCNE1 or the number of $\mathrm{PIP}_{2}$ lipids.

The differences observed between the average pore radii calculated over the MD trajectories obtained for Kv7.1 and IKs models indicate that KCNE1 may increase the tightening of the inner pore in the RC and IC states, while inducing a closer proximity between S6c region and the inner membrane surface in the AO state, which agrees with the experimental studies. The comparison of the average pore radii obtained for the $\mathrm{IKs}_{\mathrm{K}}$ models of both $4 \mathrm{PIP}_{2}$ and $8 \mathrm{PIP}_{2}$ systems with those obtained for the Kv7.1 models indicate that the MD trajectories of the IKs models of $8 \mathrm{PIP}_{2}$ system fit better the ionic conductance measures of the IKs channel (Wu et al., 2010b) compared to the Kv7.1 channel. 


\section{Discussion and Conclusion}

The present work provides tridimensional models of three states of the Kv7.1 channel in presence of KCNE1, whose features are mostly satisfying the structural constraints drawn from experimental studies. Our previous computational study of Kv7.1 models (Kasimova et al., 2015) highlighted two components of VSD-PD coupling: a protein-protein component and a protein-lipid component. Protein-protein components of this mechanism were mostly characterized by electrostatic interactions between residues from the S4-S5LINKER and residues from S6, while the protein-lipid components were characterized by state- dependent interactions between $\mathrm{PIP}_{2}$ and the $\mathrm{Kv} 7.1$ subunits.

In our IKs models, considering a second $\mathrm{PIP}_{2}$ binding site per subunit was required in order to optimize the agreement with experimental results. This work shows that in contrast to the case of $\mathrm{Kv} 7.1$, in IKs models, $\mathrm{PIP}_{2}$ lipids engage in state-independent interactions with $\mathrm{Kv} 7.1$ subunits: $\mathrm{PIP}_{2}$ intra, which is present in both $\mathrm{Kv}_{\mathrm{v}} 7.1$ and $\mathrm{IKs}$ models, predominantly binds the lower VSD basic residues, while $\mathrm{PIP}_{2}$ inter, presumably most required in IKs channels, binds the basic residues from S6 and KCNE1. In each subunit, $\mathrm{PIP}_{2}$ inter is located between CTERM regions of both the KCNE1 helix and the S6 segment (Figure S4). In 8PIP 2 IKs models (Figure S4, A, upper panel), $\mathrm{PIP}_{2}$ inter remain bound to the $\mathrm{S} 6$ residues $\mathrm{K} 362$ and $\mathrm{R} 366$ in a state independent manner, as well as to the KCNE1 residues $\mathrm{R} 67 \mathrm{~K} 69$ and $\mathrm{K} 70$ in a state dependent manner. In the $4 \mathrm{PIP}_{2} \mathrm{IK}_{s} \mathrm{AO}$ system, which is lacking the $\mathrm{PIP}_{2}$ inter (Figure S4, B, upper panel) the basic residues from S6 and from KCNE1 might repel each other and prevent $\mathrm{S} 6$ Cter from reaching $\mathrm{PIP}_{2}$ intra located in the inner membrane surface in $\mathrm{AO}$ state, as observed. 
The molecular determinants of this second $\mathrm{PIP}_{2}$ binding site we identified in our IKs models are supported by a previous functional study (Li et al., 2011) which shed light on several residues of $\mathrm{KCNE} 1$ subunit participating in $\mathrm{KCNE} 1-\mathrm{PIP}_{2}$ interactions in $\mathrm{IK}_{\text {s }}$ channels. Our results are also in agreement with an integrative study including both experimental and computational approaches (Eckey et al., 2014), which aimed at identifying Kv7.1 interactions with $\mathrm{PIP}_{2}$ in $\mathrm{IK}_{s}$ that highlighted the existence of two $\mathrm{PIP}_{2}$ binding sites for this complex, one for VSD residues and a second one for PD residues.

Noteworthy, KCNE1 residues bind both $\mathrm{PIP}_{2}$ inter and $\mathrm{PIP}_{2}$ intra in the $\mathrm{RC}$ and $\mathrm{IC}$ states which may induce a tighter packing of PD segments. Indeed, these KCNE1-PIP 2 interactions form a circle of electrostatic interactions around the cytoplasmic region of S6 (Figure 3A, B). This circle may act as a tourniquet (Figure $3 \mathrm{~A}, \mathrm{~B}$, top panels), inducing a shrinkage of the conduction pathway, leading to the lower pore radii values we obtained for IKs $\mathrm{RC}$ and IC models of $8 \mathrm{PIP}_{2}$ with respect to $\mathrm{IKs} R C$ and $I C$ models of $4 \mathrm{PIP}_{2}$, respectively. In the $A O$ models of IKs, KCNE1 loses its interactions with $\mathrm{PIP}_{2}$ intra and binds only $\mathrm{PIP}_{2}$ inter (Figure 3C). Through this interaction, one can predict that KCNE1 is pulling S6 helices towards the inner leaflet of the membrane, as $\mathrm{PIP}_{2}$ inter remains bound to $\mathrm{S} 6$ basic residues.

In the RC and IC models, the KCNE1 basic residues form electrostatic interactions with both $\mathrm{PIP}_{2}$ binding sites. This network of interactions forms a circle around S6 Cterm helices which seems to prevent those helices from moving away from the pore axis and therefore preventing the conduction pathway of the IKs channel from expending. Nevertheless, these pore radii differences between the studied IKs models estimated from the MD trajectories did not allow us to determine if these models are genuinely able to conduct potassium ions. Further studies allowing for the calculation of the free energies associated to ion translocation, beyond the scope of this work, would be required to address this issue. 
As a matter of fact, a recent computational study, which consisted in the use of machine learning methods to generate a conformational space of IKs channels, have yielded to the design of a structure-based predictor of IKs channel experimental properties including its subconductance and gating current (Ramasubramanian and Rudy, 2018b). The two sequential translations and rotations of S4 and the rotations of KCNE1 leading to VSD activation we predicted from our models are supported by the results obtained with this structure-based predictor.

The results reported by $\mathrm{Li}$ et al. (Li et al., 2011) have shown that KCNE1 increases $\mathrm{PIP}_{2}$ sensitivity 100 -fold over channels formed by the pore-forming Kv7.1 a-subunits alone. In this study the authors identified four residues (R67, K69, K70, and H73) in proximal C-terminus of KCNE1 as key determinants of $\mathrm{PIP}_{2}$ sensitivity. Mutations of these key residues in KCNE1 (R67C, R67H, K70M, and K70N) are associated with long QT syndrome (Hedley et al., 2009; Kapplinger et al., 2009; Lai et al., 2005). They reduce IKs currents and PIP2 sensitivity. Application of exogenous $\mathrm{PIP}_{2}$ to these mutants restores wild-type channel activity. The results reported in the study of $\mathrm{Li}$ et al reveal the vital role of $\mathrm{PIP}_{2}$ for $\mathrm{KCNE} 1$ modulation of IKs channels, confirming the previous studies that highlighted the inhibitory effects of $\mathrm{PIP}_{2}$ membrane depletion on $\mathrm{IK}$ s channel function, by inducing $\mathrm{PIP}_{2}$ depletion through the cotransfection of a IKs channel construct (Dahimène et al., 2006; Wang et al., 1998) and a PIP2phosphatase in Human Embryonic Kidney (HEK-293) cells (Royal et al., 2017; Suh et al., 2006). Furthermore, other studies reported that $\mathrm{PIP}_{2}$ acts as a second messenger (Logothetis et al., 2010) of various ion channels. In the case of IKs channel, PIP2 participates in the transduction of sympathetic signalling pathways induced by stress (Matavel and Lopes, 2009) or exercise (Dvir et al., 2014), leading to a left-shift on IKs voltage-dependence of activation (O-Uchi et al., 2015) and to a 2-fold increase of IKs current amplitude (Marx et al., 2002), respectively. 
The increase in $\mathrm{PIP}_{2}$ membrane levels appeared to restore the function of several loss-offunction mutations (R174C, R243C and R336Q) of KCNQ1 gene (Park et al., 2005) that are related to impaired sympathetic stimulation pathways of IKs channel (Matavel et al., 2010). Specifically, this observation suggests that both sympathetic pathways mutants activate the IKs channel by strengthening its interactions with VSD residues $\mathrm{R} 174$ and $\mathrm{R} 243$ as well as with PD residue R366. The present investigation, confirming that two of these residues are involved in specific interactions with $\mathrm{PIP}_{2}$, provides the key molecular elements that govern such a role. Indeed, our models suggest that residues $\mathrm{R} 243$ and $\mathrm{R} 366$ bind $\mathrm{PIP}_{2}$ intra and $\mathrm{PIP}_{2}$ inter in a state-dependent and a state-independent fashion, respectively. This observation agrees with the sympathetic stimulations of the VSD mutants and PD mutants cited above which yielded to different sensitivities to $\mathrm{PIP}_{2}$ membrane levels. Altogether, these results indicate a possible difference of binding affinity between both $\mathrm{PIP}_{2}$ binding sites in IKs channel.

Moreover, the family of ion channel $\beta$-subunits (KCNE1-5) contains several members that have been reported to modulate the activity of a variety of channel $\alpha$-subunits in ion channel complexes. Many of these channel $\alpha$-subunits or channel complexes are also modulated by $\mathrm{PIP}_{2}$. The KCNE1 basic residues listed above that are essential for modulation of the IKs $\mathrm{PIP}_{2}$ sensitivity are highly conserved across all members of the KCNE family of peptides (Figure 4), suggesting that modulation of $\mathrm{PIP}_{2}$ sensitivity may be a common mechanism of current modulation by the KCNE $\beta$-subunits. As we built models of the transmembrane regions of both $\mathrm{Kv}_{\mathrm{v}}$.1 and KCNE1 subunits, the recently reported $\mathrm{PIP}_{2}$ binding sites, located in the cytoplasmic region of the Kv7.1 (Tobelaim et al., 2017) and the Kv7.3 subunits (Choveau et al., 2018), could not have been addressed in the frame of this study. 
The present analysis demonstrated the coherence of the simulation of our IKs models in a system that contain eight $\mathrm{PIP}_{2}$ molecules instead of four, each located in the respective binding sites of Kv7.1 and KCNE1 subunits, in order to fit the experimental data as much as possible. Hence, the robustness of the resulting MD trajectories will be used for ongoing work that features the investigation of the molecular determinants of the VSD-PD coupling and pore opening mechanisms of the Kv7.1 channel, in both the absence (Hou et al., 2020) and the presence of the KCNE1 ancillary subunit. 


\section{Material and Methods}

To investigate the structural determinants of $\mathrm{PIP}_{2}$ binding sites of the IKs channel, we first needed to build molecular models which had to be as trustworthy as possible with respect to experimental data available. Since no high-resolution structure of the Kv7.1 channel was available yet, we used homology modeling to build several models of the channel embedded within a lipid bilayer (Page et al., 2007). For Kv7.1, the activation mechanism involves three stable states, i.e., RC, IC, and AO states. Accordingly, each state was modeled to obtain a larger spectrum of possible a-subunits conformations, allowing for the prediction of possible transition mechanisms for IKs channel. For these models, the NTERM and CTERM cytoplasmic regions of Kv7.1 were ignored. Only residues 122 to 366 from KCNQ1 human sequence, corresponding to the transmembrane region of the channel, were considered. To fully characterize the modulation of $\mathrm{Kv} 7.1$ by the KCNE1 subunits, we built each state model along with the TMD (residues 39 to 76 ) of the human KCNE1 NMR structure (Tian et al., 2007) using a Kv7.1:KCNE1 subunit ratio of $4: 4$.

- Homology modeling of Kv7.1 in its distinct states

Homology modeling, also known as comparative modeling, aims at building a protein structure from its primary sequence, starting from the premise that two proteins with similar primary sequences will be displaying similar folds (3D structures). To adjust the salt-bridge patterns of the VSD with respect to experimental results (Wu et al., 2010b, 2010a), and thereby obtain distinct activation state models of the VSD, the charged group of E160 (E1) was constrained to be in close proximity with:

- R237 (R4) in activated/open (AO) model, using the refined crystallographic structure of Kv1.2 (Chen et al., 2010) as a template 
- R231 (R2) in Intermediate/Closed (IC) model using the y conformation of the Kv1.2 subunit refined structure obtained from previous MD simulations (Delemotte et al., 2011, 2015)

- R228 (R1) in Resting/Closed (RC) model, using the $\varepsilon$ conformation of Kv1.2 refined structure obtained from the aforementioned study.

The alignment of the $\mathrm{Kv} 7.1$ human sequence and $\mathrm{Kv1.2}$ rat sequence was first conducted automatically, using ClustalW2 (Larkin et al., 2007). For the PD, the percentage of sequence identity between Kv7.1 and Kv1.2 is $36 \%$. For the VSD, the percentage is $19.5 \%$. This lower sequence identity is mainly due to the S2-S3 loop, which is longer in Kv7.1 sequence than in the one of Kv1.2. To overcome this discrepancy, this alignment was refined manually and locally. Indeed, we specifically aligned Kv7.1 important residues (conserved acidic residue of S2, conserved CTC, S4 conserved gating charges) with similar Kv1.2 ones. Plus, insertions and deletions were concentrated in the loop regions. Eventually, without S2-S3 loop, the percentage of identity between $\mathrm{Kv} 7.1$ and $\mathrm{Kv} 1.2$ was increased to $25 \%$ for the VSD.

For each stable state of Kv7.1, fifty models were generated using MODELLER (Eswar et al., 2007). This software performs constrained modeling, a technique which consists in using template coordinates and sequence alignment information as constraints for the building of 3D models. S2-S3 loop has been modeled from a template 3D structure extracted from NMR data (Peng et al., 2014), which suggests a helical structure for this connecting loop. Since MODELLER allows one to add specific geometric restraints, several ones were applied according to site-mutagenesis results to increase the reliability of these models with respect to experimental data. Other constraints drawn from 3D models of IKs channel stable states (Kang et al., 2008) were also used to predict the position of KCNE1 subunits with respect to Kv7.1. 
Among these 50 obtained models, the best 10 were selected according to their potential energy values, calculated using DOPE (ㅁiscrete ㅇptimizing Protein Energy) (Shen and Sali, 2006) knowledge-based scoring function, implemented in MODELLER. The stereochemical quality of these models were evaluated using PROCHECK software (Laskowski et al., 1993). For each stable state, the structure presenting the highest number of Phi and Psi torsion angles in Ramachandran's plot well favored areas (>95\%), as well as the lowest number of torsion angles in the disfavored areas $(<5 \%)$, was chosen to perform molecular dynamics simulations to study our models.

\section{- Molecular Dynamics simulations}

To reproduce the behavior of IKs channels in their natural environment, the three models were embedded in lipid bilayers prior to the simulations. To do this, we used a method available in the input generator of CHARMM (Jo et al., 2008), which consists in adding lipid molecules around the protein structure (Wu et al., 2014). Since Phosphatidylcholine (PC) lipids are the most abundant lipids found in cell membranes, Palmitoyl-Oleyl PC (POPC) lipids were selected to build the bilayer in each of our nine systems. To incorporate phospholipids in the correct binding sites of the channels, a $\mathrm{PIP}_{2}$ molecule was added within the inner leaflet of the bilayer, at the bottom of each VSD ( $\mathrm{PIP}_{2}$ intra) with respect to experimental studies (Eckey et al., 2014; Zaydman et al., 2013) and computational results of MD simulations conducted on Kv7.1 subunits along with $\mathrm{PIP}_{2}$ (Kasimova et al., 2015; Zaydman et al., 2014). We embedded the RC, IC and AO states models in two distinct environments. In the first system, referred to as $4 \mathrm{PIP}_{2}$, we added only $\mathrm{PIP}_{2}$ intra, corresponding to the binding site of Kv7.1 subunits. In the second system, referred to as 8PIP 2 we added a second $\mathrm{PIP}_{2}$ molecule at the bottom of KCNE1 subunits, following data from experiments that highlighted a second $\mathrm{PIP}_{2}$ binding site in IKs channels (Li et al., 2011) (cf. Figure 1.) 
Simulations were carried out using the NPT ensemble for the equilibration of the systems, at $300 \mathrm{~K}$, and $1 \mathrm{~atm}$. (Noteworthy, POPC has a transition temperature above $300 \mathrm{~K}$, i.e. it is in its liquid crystal phase). Chemical bond lengths between hydrogens and heavy atoms were all constrained at their equilibrium values so that a time-step of 2.0 fs could be used. The systems (lipids+channel) were surrounded by a $150 \mathrm{mM}[\mathrm{KCl}]$ solution. We used the CHARMM36 force-field (Huang and Mackerell, 2013), along with CMAP correction (Buck et al., 2006) and NAMD code (Phillips et al., 2005) to perform all MD calculations.

The MD simulations were conducted in four steps, during which motion constraints were applied on the whole system, and then gradually released. The first step of 200 ps aimed at fully solvating the protein in the membrane, by letting water molecules rearrange themselves around the protein. Accordingly, constraints were set up on all IKs atoms. The second step of 6 ns was run to relax the side chains of the protein, so the constrains were kept only on IKs backbone. During these two steps, the positions of $\mathrm{PIP}_{2}$ phosphorus atoms of in the system were kept constant, to maintain this lipid in its correct binding sites. The third step was conducted to allow $\mathrm{PIP}_{2}$ lipids to rearrange around the protein complex and within the lipid bilayer. Hence the constraints on $\mathrm{PIP}_{2}$ phosphorus atoms were removed but we maintained on the backbone of IKs complex. This step of 70 ns was also conducted to let the density of the system reach a constant value. Finally, the last step corresponds to the so-called production phase. This step, performed without any specific forces on any coordinate of the system, lasts approximately 500 ns. Only the backbone of the selectivity filter (corresponding to the voltage-gated ion channel conserved 311-TTIGYG-316 sequence) was spatially constrained, to prevent ion conduction during the simulations. 
- Validation of IKs models by MD simulation analyses: Strategy

We considered here only the production phase of MD simulations. To assess the reliability of the models with respect to experimental data, we confronted, for each state of the IKs channel, the 2 systems against a set of results obtained from biophysical characterization. To achieve this, we first gathered all the cross-linking studies conducted on IKs channel to identify: (i) The VSD salt-bridge patterns, (ii) The Kv7.1 intersubunit and intrasubunit neighbor residues, (iii) The KCNE1/Kv7.1 neighbor residues and (iv) The Kv7.1 and KCNE1 residues which bind $\mathrm{PIP}_{2}$. Cysteine cross-linking studies assume that mutated cysteine residues can form disulfide bonds with a significant formation rate constant if the distance between their $\mathrm{C}_{\beta}$ atoms is $\leqslant 13.2 \AA$ (Careaga and Falke, 1992). Accordingly, we evaluated the $\mathrm{C}_{\beta}-\mathrm{C}_{\beta}$ distances of each pair of residues in the MD trajectories we obtained for our IKs models and reported their average values (see tables S2-S3 (Supporting Information)).

A pair was considered as fulfilled in the model if the average distance between the $\mathrm{C}_{\beta}$ atoms pair was below $\sim 13 \AA$ for at least three channel subunits out of four. These distances were monitored every 2 ns over the MD trajectories, using a designed program written in TCL language which we executed within the scripting interface of Visual Molecular Dynamics (VMD) software (Humphrey et al., 1996). We investigated the electrostatic interactions and the hydrogen bonds that are expected between the $S 4$ gating charges and the $S 2$ negative ones (Table S1), as well as the ones between basic side chains from the cytoplasmic region of KCNE1 and $\mathrm{PIP}_{2}$ (Table S4). These were monitored every 2 ns and were considered satisfied if the average distance between the respective charge moieties were below $3.5 \AA$, which is the average distance encountered for ion pairs in protein NMR structures (Kumar and Nussinov, 2002). 
For these calculations, we considered that the positive charge of arginine side chains is delocalized between three terminal nitrogen atoms of its guanidium group, and the negative charges of glutamate and aspartate side chains are shared by two oxygen atoms from their respective carboxyl group. For $\mathrm{PIP}_{2}$, its five negative charges are delocalized between eight oxygen atoms from its three phosphate groups (Figure S2). Hence, the salt bridges they form with the channel residues were monitored by computing each of the atom pair combinations of terminal nitrogen atoms from Arg guanidium group and terminal oxygens from Glu or Asp carboxyl groups. The distance graphics were designed using $\mathrm{R}$ scripting language (Heiner Schwarte et al., 2017). For each pair of residues, an interaction was considered present if found in at least three subunits out of four.

Starting from the premise that pore radius of open $\mathrm{Kv}_{v}$ channels gates energetically favors ionic conduction (Beckstein et al., 2004; Peter and Hummer, 2005; Treptow and Tarek, 2006), one expects that channel models with larger pore radii are likely to facilitate ion conduction and therefore generate similar ionic currents as those recorded experimentally. Pore radii and pore solvent accessible surfaces of all models were calculated and generated every 20 ns of their respective MD trajectory, both using HOLE program (Smart et al., 1996). For each model, we reported the average pore radii values along the pore axis, i.e. the conduction pathway. For each state, we compared the obtained pore radii with those calculated for the Kv7.1 models built in the frame of a previous study. Indeed, KCNE1 is known to enhance Kv7.1 ionic currents in the AO state, while abolishing this current in both the Intermediate and the RC states. Pore surfaces were rendered with VMD.

For each IKs state, the MD trajectory which presented the highest number of salt bridges in agreement with experimental data, and the most relevant average pore radii with respect to those obtained for the Kv7.1 models, was selected as the best model of IKs channel complex. 


\section{References}

Barhanin, J., Lesage, F., Guillemare, E., Fink, M., Lazdunski, M., and Romey, G. (1996). KvLQT1 and IsK (minK) proteins associate to form the IKS cardiac potassium current. Nature 384, 78-80.

Beckstein, O., Tai, K., and Sansom, M.S.P. (2004). Not ions alone: Barriers to ion permeation in nanopores and channels. J. Am. Chem. Soc. 126, 14694-14695.

Buck, M., Bouguet-Bonnet, S., Pastor, R.W., and MacKerell, A.D. (2006). Importance of the CMAP correction to the CHARMM22 protein force field: Dynamics of hen lysozyme. Biophys. J. 90, L36-L38.

Careaga, C.L., and Falke, J.J. (1992). Thermal Motions of Surface $\alpha$-Helices in the D-Galactose Chemosensory Receptor. J. Mol. Biol. 226, 1219-1235.

Catterall, W.A. (1986). Molecular Properties of Voltage-Sensitive Sodium Channels. New Insights into Cell Membr. Transp. Process. 3-20.

Chen, X., Wang, Q., Ni, F., and Ma, J. (2010). Structure of the full-length Shaker potassium channel Kv1.2 by normal-mode-based X-ray crystallographic refinement. Proc. Natl. Acad. Sci. U. S. A. 107, 11352-11357.

Choveau, F.S., De la Rosa, V., Bierbower, S.M., Hernandez, C.C., and Shapiro, M.S. (2018). Phosphatidylinositol 4,5-bisphosphate (PIP 2) regulates KCNQ3 K + channels by interacting with four cytoplasmic channel domains. J. Biol. Chem. 293, 19411-19428.

Dahimène, S., Alcoléa, S., Naud, P., Jourdon, P., Escande, D., Brasseur, R., Thomas, A., Baró, I., and Mérot, J. (2006). The N-terminal juxtamembranous domain of KCNQ1 is critical for channel surface expression: Implications in the Romano-Ward LQT1 syndrome. Circ. Res. 99, 1076-1083.

Delemotte, L., Tarek, M., Klein, M.L., Amaral, C., and Treptow, W. (2011). Intermediate states of the Kv1.2 voltage sensor from atomistic molecular dynamics simulations. Proc. Natl. Acad. Sci. 108, 6109-6114.

Delemotte, L., Kasimova, M.A., Klein, M.L., Tarek, M., and Carnevale, V. (2015). Free-energy landscape of ionchannel voltage-sensor-domain activation. Proc. Natl. Acad. Sci. 112, 124-129.

Díaz-Franulic, I., Sepúlveda, R. V., Navarro-Quezada, N., González-Nilo, F., and Naranjo, D. (2015). Pore dimensions and the role of occupancy in unitary conductance of Shaker K channels. J. Gen. Physiol. 146, 133146.

Durell, S.R., and Guy, H.R. (1992). Atomic scale structure and functional models of voltage-gated potassium channels. Biophys. J. 62, 238-250.

Dvir, M., Strulovich, R., Sachyani, D., Ben-Tal Cohen, I., Haitin, Y., Dessauer, C., Pongs, O., Kass, R., Hirsch, J.A., Attali, B., et al. (2014). Long QT mutations at the interface between KCNQ1 helix C and KCNE1 disrupt IKS regulation by PKA and PIP2. J. Cell Sci. 127, 3943-3955.

Eckey, K., Wrobel, E., Strutz-Seebohm, N., Pott, L., Schmitt, N., and Seebohm, G. (2014). Novel Kv7.1Phosphatidylinositol 4,5-Bisphosphate Interaction Sites Uncovered by Charge Neutralization Scanning. J. Biol. Chem. 289, 22749-22758.

Eswar, N., Webb, B., Marti-Renom, M.A., Madhusudhan, M.S., Eramian, D., Shen, M.-Y., Pieper, U., and Sali, A. (2007). Comparative Protein Structure Modeling Using MODELLER. Curr. Protoc. Protein Sci. 50, 2.9.12.9.31.

Gofman, Y., Shats, S., Attali, B., Haliloglu, T., and Ben-Tal, N. (2012). How Does KCNE1 Regulate the Kv7.1 Potassium Channel? Model-Structure, Mutations, and Dynamics of the Kv7.1-KCNE1 Complex. Structure 20, 1343-1352. 
Hedley, P.L., Jørgensen, P., Schlamowitz, S., Wangari, R., Moolman-Smook, J., Brink, P.A., Kanters, J.K., Corfield, V.A., and Christiansen, M. (2009). The genetic basis of long QT and short QT syndromes: A mutation update. Hum. Mutat. 30, 1486-1511.

Heiner Schwarte, Guido Masarotto, Seth Falcon, Douglas Bates, John Chambers, Peter Dalgaard, Robert Gentleman, Kurt Hornik, Ross Ihaka, Tomas Kalibera, et al. (2017). R: A language and environment for statistical computing. (Vienna, Austria: R Foundation for Statistical Computing).

Hilgemann, D.W., Feng, S., and Nasuhoglu, C. (2001). The Complex and Intriguing Lives of PIP2 with Ion Channels and Transporters. Sci. STKE Signal Transduct. Knowl. Environ. 2001, re19-re19.

Hou, P., Eldstrom, J., Shi, J., Zhong, L., McFarland, K., Gao, Y., Fedida, D., and Cui, J. (2017). Inactivation of KCNQ1 potassium channels reveals dynamic coupling between voltage sensing and pore opening. Nat. Commun. 8.

Hou, P., Kang, P.W., Kongmeneck, A.D., Yang, N.-D., Liu, Y., Shi, J., Xu, X., White, K.M., Zaydman, M.A., Kasimova, M.A., et al. (2020). Two-stage electro-mechanical coupling of a KV channel in voltage-dependent activation. Nat. Commun. 11, 676.

Huang, J., and Mackerell, A.D. (2013). CHARMM36 all-atom additive protein force field: Validation based on comparison to NMR data. J. Comput. Chem. 34, 2135-2145.

Humphrey, W., Dalke, A., and Schulten, K. (1996). VMD: Visual molecular dynamics. J. Mol. Graph. 14, 33-38.

Jalily Hasani, H., Ahmed, M., and Barakat, K. (2017). A comprehensive structural model for the human KCNQ1/KCNE1 ion channel. J. Mol. Graph. Model. 78, $26-47$.

Jalily Hasani, H., Ganesan, A., Ahmed, M., and Barakat, K.H. (2018). Effects of protein-protein interactions and ligand binding on the ion permeation in KCNQ1 potassium channel. PLoS One 13, e0191905.

Jo, S., Kim, T., lyer, V.G., and Im, W. (2008). CHARMM-GUI: A web-based graphical user interface for CHARMM. J. Comput. Chem. 29, 1859-1865.

Kang, C., Tian, C., Sönnichsen, F.D., Smith, J.A., Meiler, J., George, A.L., Vanoye, C.G., Kim, H.J., and Sanders, C.R. (2008). Structure of KCNE1 and Implications for How It Modulates the KCNQ1 Potassium Channel † ‡. Biochemistry 47, 7999-8006.

Kapplinger, J.D., Tester, D.J., Salisbury, B.A., Carr, J.L., Harris-Kerr, C., Pollevick, G.D., Wilde, A.A.M., and Ackerman, M.J. (2009). Spectrum and prevalence of mutations from the first 2,500 consecutive unrelated patients referred for the FAMILION® long QT syndrome genetic test. Hear. Rhythm 6, 1297-1303.

Kasimova, M.A., Zaydman, M.A., Cui, J., and Tarek, M. (2015). PIP2-dependent coupling is prominent in Kv7.1 due to weakened interactions between S4-S5 and S6. Sci. Rep. 5, 7474.

Kumar, S., and Nussinov, R. (2002). Relationship between ion pair geometries and electrostatic strengths in proteins. Biophys. J. 83, 1595-1612.

Lacroix, J.J., and Bezanilla, F. (2011). Control of a final gating charge transition by a hydrophobic residue in the S2 segment of a K+ channel voltage sensor. Proc. Natl. Acad. Sci. U. S. A. 108, 6444-6449.

Lai, L.-P., Su, Y.-N., Chiang, F.-T., Juang, J.-M., Liu, Y.-B., Ho, Y.-L., Chen, W.-J., Yeh, S.-J., Wang, C.-C., Ko, Y.-L., et al. (2005). Denaturing high-performance liquid chromatography screening of the long QT syndromerelated cardiac sodium and potassium channel genes and identification of novel mutations and single nucleotide polymorphisms. J. Hum. Genet. 50, 490-496.

Larkin, M.A.A., Blackshields, G., Brown, N.P.P., Chenna, R., Mcgettigan, P.A.A., McWilliam, H., Valentin, F., Wallace, I.M.M., Wilm, A., Lopez, R., et al. (2007). Clustal W and Clustal X version 2.0. Bioinformatics 23, 29472948. 
Laskowski, R.A., MacArthur, M.W., Moss, D.S., and Thornton, J.M. (1993). PROCHECK: a program to check the stereochemical quality of protein structures. J. Appl. Crystallogr. 26, 283-291.

Li, Y., Gamper, N., Hilgemann, D.W., and Shapiro, M.S. (2005). Regulation of Kv7 (KCNQ) K+ Channel Open Probability by Phosphatidylinositol 4,5-Bisphosphate. J. Neurosci. 25, 9825-9835.

Li, Y., Zaydman, M.A., Wu, D., Shi, J., Guan, M., Virgin-Downey, B., and Cui, J. (2011). KCNE1 enhances phosphatidylinositol 4,5-bisphosphate (PIP2) sensitivity of IKs to modulate channel activity. Proc. Natl. Acad. Sci. U. S. A. 108, 9095-9100.

Liin, S.I., Barro-Soria, R., and Larsson, H.P. (2015). The KCNQ1 channel - remarkable flexibility in gating allows for functional versatility. J. Physiol. 593, 2605-2615.

Logothetis, D.E., Petrou, V.I., Adney, S.K., and Mahajan, R. (2010). Channelopathies linked to plasma membrane phosphoinositides. Pflugers Arch. Eur. J. Physiol. 460, 321-341.

Marx, S.O., Kurokawa, J., Reiken, S., Motoike, H., D’Armiento, J., Marks, A.R., and Kass, R.S. (2002). Requirement of a macromolecular signaling complex for $\beta$ adrenergic receptor modulation of the KCNQ1KCNE1 potassium channel. Science (80-. ). 295, 496-499.

Matavel, A., and Lopes, C.M.B. (2009). PKC activation and PIP2 depletion underlie biphasic regulation of IKs by Gq-coupled receptors. J. Mol. Cell. Cardiol. 46, 704-712.

Matavel, A., Medei, E., and Lopes, C.M.B. (2010). PKA and PKC partially rescue Long QT type 1 phenotype by restoring channel-PIP 2 interactions. Channels 4, 3-11.

McCrossan, Z.A., and Abbott, G.W. (2004). The MinK-related peptides. Neuropharmacology 47, 787-821.

Murray, C.I., Westhoff, M., Eldstrom, J., Thompson, E., Emes, R., and Fedida, D. (2016). Unnatural amino acid photo-crosslinking of the IKs channel complex demonstrates a KCNE1:KCNQ1 stoichiometry of up to 4:4. Elife 5.

Nakajo, K., Ulbrich, M.H., Kubo, Y., and Isacoff, E.Y. (2010). Stoichiometry of the KCNQ1 - KCNE1 ion channel complex. Proc. Natl. Acad. Sci. U. S. A. 107, 18862-18867.

Napolitano, C., Priori, S.G., Schwartz, P.J., Bloise, R., Ronchetti, E., Nastoli, J., Bottelli, G., Cerrone, M., and Leonardi, S. (2005). Genetic testing in the long QT syndrome: Development and validation of an efficient approach to genotyping in clinical practice. J. Am. Med. Assoc. 294, 2975-2980.

Nerbonne, J.M., and Kass, R.S. (2005). Molecular physiology of cardiac repolarization. Physiol. Rev. 85, 12051253.

O-Uchi, J., Rice, J.J., Ruwald, M.H., Parks, X.X., Ronzier, E., Moss, A.J., Zareba, W., and Lopes, C.M. (2015). Impaired IKs channel activation by $\mathrm{Ca2}+$-dependent PKC shows correlation with emotion/arousal-triggered events in LQT1. J. Mol. Cell. Cardiol. 79, 203-211.

Page, R.C., Li, C., Hu, J., Gao, F.P., and Cross, T.A. (2007). Lipid bilayers: an essential environment for the understanding of membrane proteins. Magn. Reson. Chem. 45, S2--S11.

Park, K.-H.H., Piron, J., Dahimene, S., Mérot, J., Baró, I., Escande, D., Loussouarn, G., Mérot, J., Baró, I., Escande, D., et al. (2005). Impaired KCNQ1-KCNE1 and phosphatidylinositol-4,5-bisphosphate interaction underlies the long QT syndrome. Circ. Res. 96, 730-739.

Peng, D., Kim, J.-H.H., Kroncke, B.M., Law, C.L., Xia, Y., Droege, K.D., Van Horn, W.D., Vanoye, C.G., and Sanders, C.R. (2014). Purification and Structural Study of the Voltage-Sensor Domain of the Human KCNQ1 Potassium Ion Channel. Biochemistry 53, 2032-2042.

Peter, C., and Hummer, G. (2005). Ion Transport through Membrane-Spanning Nanopores Studied by Molecular Dynamics Simulations and Continuum Electrostatics Calculations. Biophys. J. 89, 2222-2234. 
Phillips, J.C., Braun, R., Wang, W., Gumbart, J., Tajkhorshid, E., Villa, E., Chipot, C., Skeel, R.D., Kalé, L., and Schulten, K. (2005). Scalable molecular dynamics with NAMD. J. Comput. Chem. 26, 1781-1802.

Ramasubramanian, S., and Rudy, Y. (2018a). The Structural Basis of IKs Ion Channel Activation: Mechanistic Insights from Molecular Simulations. Biophys. J. 114, 2584-2594.

Ramasubramanian, S., and Rudy, Y. (2018b). The Structural Basis of IKs Ion-Channel Activation: Mechanistic Insights from Molecular Simulations. Biophys. J. 114, 2584-2594.

Roux, B. (2006). Dissecting the Coupling between the Voltage Sensor and Pore Domains. Neuron 52, 568569.

Royal, A.A., Tinker, A., and Harmer, S.C. (2017). Phosphatidylinositol-4,5-bisphosphate is required for KCNQ1/KCNE1 channel function but not anterograde trafficking. PLoS One 12.

Shen, M., and Sali, A. (2006). Statistical potential for assessment and prediction of protein structures. Protein Sci. 15, 2507-2524.

Smart, O.S., Neduvelil, J.G., Wang, X., Wallace, B.A., and Sansom, M.S.P. (1996). HOLE: A program for the analysis of the pore dimensions of ion channel structural models. J. Mol. Graph. 14, 354-360.

Splawski, I., Shen, J., Timothy, K.W., Lehmann, M.H., Priori, S., Robinson, J.L., Moss, A.J., Schwartz, P.J., Towbin, J.A., Vincent, G.M., et al. (2000). Spectrum of Mutations in Long-QT Syndrome Genes. Circulation 102, $1178-1185$.

Suh, B.C., Inoue, T., Meyer, T., and Hille, B. (2006). Rapid chemically induced changes of Ptdlns(4,5)P2 gate KCNQ ion channels. Science (80-. ). 314, 1454-1457.

Sun, J., and MacKinnon, R. (2017). Cryo-EM Structure of a KCNQ1/CaM Complex Reveals Insights into Congenital Long QT Syndrome. Cell 169, 1042-1050.

Sun, J., and MacKinnon, R. (2020). Structural Basis of Human KCNQ1 Modulation and Gating. Cell 180, 340347.e9.

Tao, X., Lee, A., Limapichat, W., Dougherty, D.A., and MacKinnon, R. (2010). A gating charge transfer center in voltage sensors. Science (80-. ). 328, 67-73.

Tian, C., Vanoye, C.G., Kang, C., Welch, R.C., Kim, H.J., George, A.L., Sanders, C.R., Hak, J.K., George, A.L., Sanders, C.R., et al. (2007). Preparation, Functional Characterization, and NMR Studies of Human KCNE1, a Voltage-Gated Potassium Channel Accessory Subunit Associated with Deafness and Long QT Syndrome †, ‡. Biochemistry 46, 11459-11472.

Tobelaim, W.S., Dvir, M., Lebel, G., Cui, M., Buki, T., Peretz, A., Marom, M., Haitin, Y., Logothetis, D.E., Hirsch, J.A., et al. (2017). Ca 2+-Calmodulin and PIP2 interactions at the proximal C-terminus of Kv7 channels. Channels 11, 686-695.

Treptow, W., and Tarek, M. (2006). Molecular restraints in the permeation pathway of ion channels. Biophys. J. 91, L26--28.

Tristani-Firouzi, M., and Sanguinetti, M.C. (1998). Voltage-dependent inactivation of the human K + channel KvLQT1 is eliminated by association with minimal $\mathrm{K}+$ channel (minK) subunits. J. Physiol. 510, 37-45.

Wang, W., Xia, J., and Kass, R.S. (1998). MinK-KvLQT1 fusion proteins, evidence for multiple stoichiometries of the assembled I(sK) channel. J. Biol. Chem. 273, 34069-34074.

Wiener, R., Haitin, Y., Shamgar, L., Fernández-Alonso, M.C., Martos, A., Chomsky-Hecht, O., Rivas, G., Attali, B., and Hirsch, J.A. (2008). The KCNQ1 (Kv7.1) COOH terminus, a multitiered scaffold for subunit assembly and protein interaction. J. Biol. Chem. 283, 5815-5830. 
Wu, D., Delaloye, K., Zaydman, M.A., Nekouzadeh, A., Rudy, Y., and Cui, J. (2010a). State-dependent electrostatic interactions of S4 arginines with E1 in S2 during Kv7.1 activation. J. Gen. Physiol. 135, 595-606.

Wu, D., Pan, H., Delaloye, K., and Cui, J. (2010b). KCNE1 Remodels the Voltage Sensor of Kv7.1 to Modulate Channel Function. Biophys. J. 99, 3599-3608.

Wu, E.L., Cheng, X., Jo, S., Rui, H., Song, K.C., Dávila-Contreras, E.M., Qi, Y., Lee, J., Monje-Galvan, V., Venable, R.M., et al. (2014). CHARMM-GUI Membrane Builder toward realistic biological membrane simulations. J. Comput. Chem. 35, 1997-2004.

Xu, Y., Wang, Y., Meng, X.-Y.Y., Zhang, M., Jiang, M., Cui, M., and Tseng, G.-N.N. (2013). Building KCNQ1/KCNE1 Channel Models and Probing their Interactions by Molecular-Dynamics Simulations. Biophys. J. 105, 2461-2473.

Zaydman, M.A., and Cui, J. (2014). PIP2 regulation of KCNQ channels: biophysical and molecular mechanisms for lipid modulation of voltage-dependent gating. Front. Physiol. 5, 195.

Zaydman, M.A., Silva, J.R., Delaloye, K., Li, Y., Liang, H., Larsson, H.P., Shi, J., and Cui, J. (2013). Kv7.1 ion channels require a lipid to couple voltage sensing to pore opening. Proc. Natl. Acad. Sci. 110, 13180-13185.

Zaydman, M.A., Kasimova, M.A., McFarland, K., Beller, Z., Hou, P., Kinser, H.E., Liang, H., Zhang, G., Shi, J., Tarek, M., et al. (2014). Domain-domain interactions determine the gating, permeation, pharmacology, and subunit modulation of the IKs ion channel. Elife 3, e03606. 
bioRxiv preprint doi: https://doi. org/101101/2021 01.13.426035; this version posted January 13, 2021. The copyright holder for this preprint (which was not certified by peer review) is the author/funder, who has granted bioRxiv a license to display the preprint in perpetuity. It is made available under aCC-BY-NC-ND 4.0 International license.

\section{Figures}

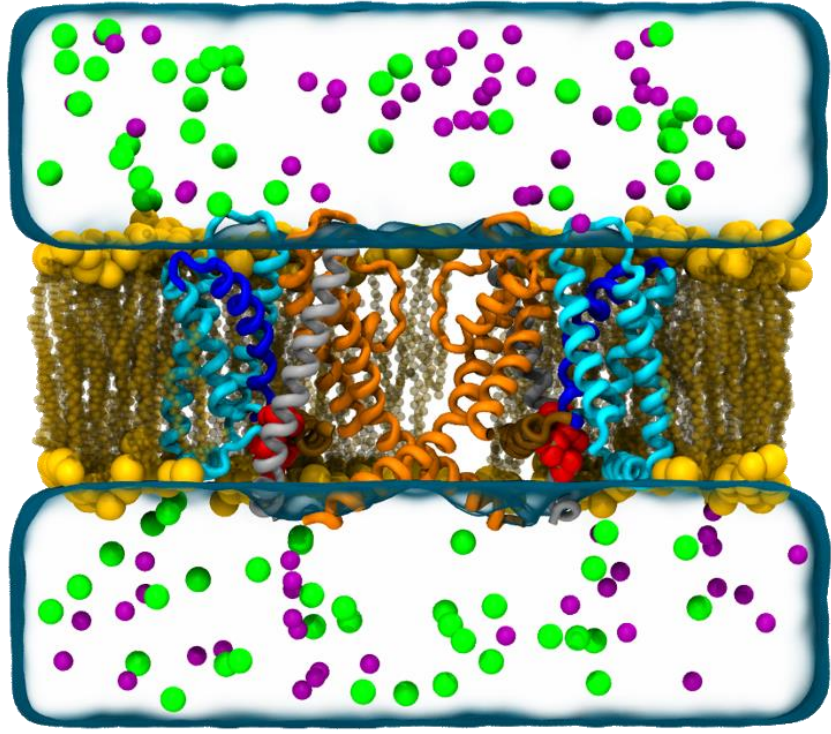

$\mathrm{IK}_{\mathrm{s}}: 4 \mathrm{PIP}_{2}$

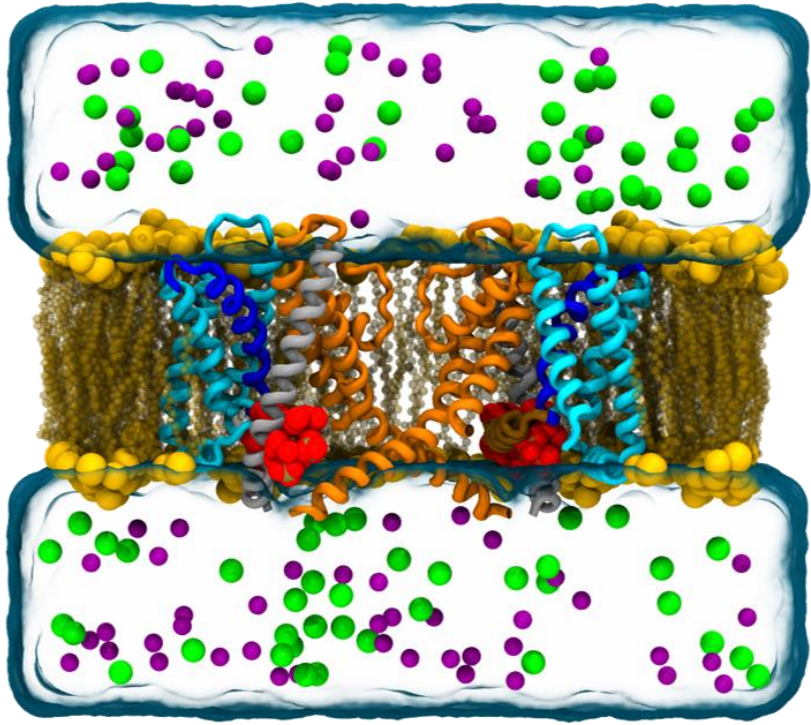

$\mathrm{IK}_{\mathrm{s}}: 8 \mathrm{PIP}_{2}$

Figure 1: MD systems models of IKs models.

Representation of Kv7.1 models in absence (left) and presence (right) of KCNE1 (gray ribbons) and embedded in a POPC membrane (in yellow spheres) with $\mathrm{PIP}_{2}$ lipids (in red spheres). Two $\mathrm{K}_{\mathrm{v}} 7.1$ subunits are shown for clarity. Transmembrane segments are represented in ribbons. VSD segments are colored in cyan, S4 in blue, S4-S5LINKER in brown and PD segments in orange. Membrane-embedded models are surrounded by water (in transparent blue surface) as well as $\mathrm{K}^{+}$(purple spheres) and $\mathrm{Cl}^{-}$(green spheres) ions. 

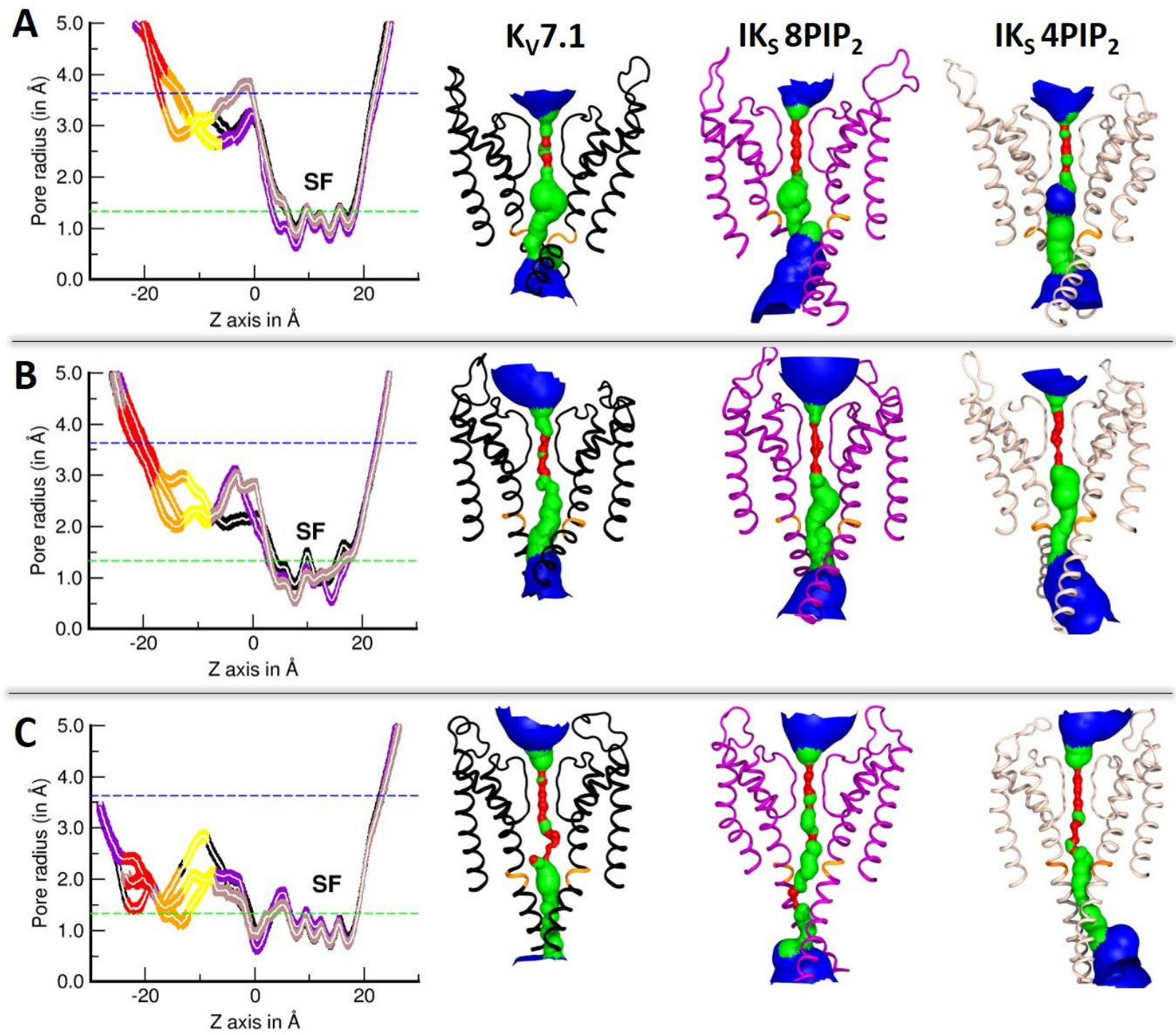

Figure 2: Validation of $I \mathrm{~K}_{\mathrm{S}}$ models through pore radii calculations

The graphs report the average pore radii (left) along the conduction pathway of $\mathrm{K}_{\mathrm{v}} 7.1$ (black curves) IKs $8 \mathrm{PIP}_{2}$ (purple curves) and IKs 4PIP 2 (beige curves) models in A. AO state $\mathbf{B}$. Intermediate state and $\mathbf{C}$. RC state. Averages radii at the levels of residues G345, S349 and L353 are depicted in yellow, orange and red curves, respectively. The right panels show cartoon representations of Kv7.1 (black) IKs $8 \mathrm{PIP}_{2}$ (purple) and IKs $4 \mathrm{PIP}_{2}$ (beige) models. Potassium ionic $(1.33 \AA$ ) and hydrodynamic radii $(3.6 \AA)$ are represented by green and blue dashed lines in the graphs, respectively. Pore solvent accessible surfaces are colored as follows. Pore radii values inferior to $\mathrm{K}+$ ionic radius, are colored in red. Pore radii values ranging between $\mathrm{K}+$ ionic radius and $\mathrm{K}+$ hydrodynamic radius, are colored in green. Pore radii values superior to $\mathrm{K}+$ hydrodynamic radius, are colored in blue. 

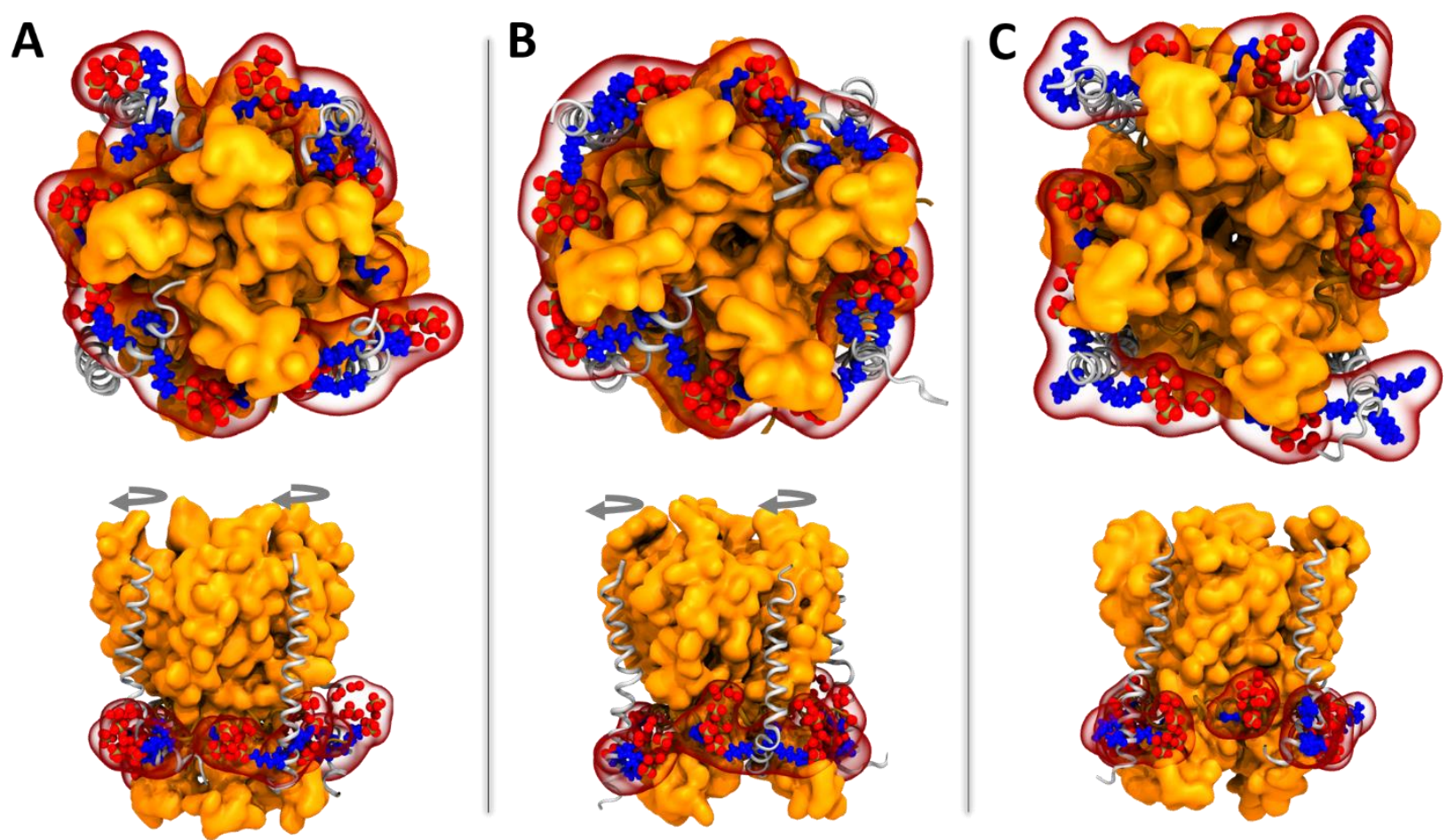

Figure 3: Structural mapping of KCNE1 basic residues and $\mathrm{PIP}_{2}$ lipids in the pore domain of $\mathrm{IK}_{\mathrm{S}}$ models in $8 \mathrm{PIP}_{2}$ systems

Intracellular view (top panels) and side view (bottom panels) of $\mathrm{Kv}_{\mathrm{v}} 7.1$ pore domain segments (in orange surface) along with S4-S5LINKER (in brown ribbons), KCNE1 subunits (in gray ribbons) and $\mathrm{PIP}_{2}$ phosphate groups (in red spheres) in A. RC model, B. IO model and C. AO model. KCNE1 basic residues R67, K69 and K70 are shown in blue spheres. S4-S5 LINKER residue R249 that interact with $\mathrm{PIP}_{2}$ intra in a state independent fashion is shown in blue sticks. The successive rotational movements of KCNE1 subunits predicted to occur during RC-IC and IC-AO transitions are shown in gray circled arrows.

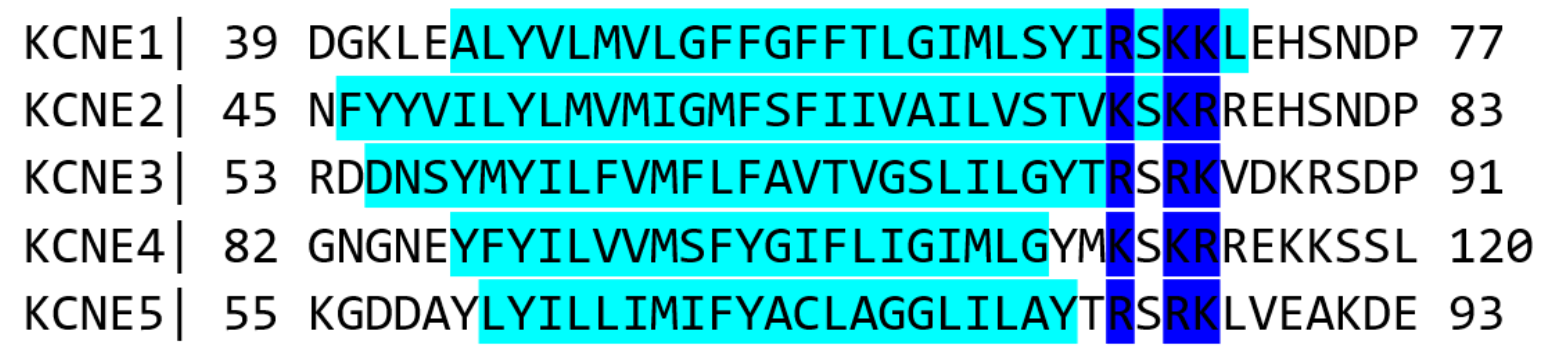

Figure 4: Conserved basic residues in the of KCNE ancillary subunits.

The picture shows a sequence alignment of the transmembrane domains (TMD) of KCNE subunits, highlighted in cyan. The conserved basic residues, located at the end of the TMD, near their CTERM domains, are highlighted in blue. 


\section{Modulation of the $\mathrm{IK}_{\mathrm{S}}$ channel by $\mathrm{PIP}_{2}$ require two binding sites per monomer}

Audrey Deyawe Kongmeneck ${ }^{1}$, Marina A. Kasimova ${ }^{1}$, and Mounir Tarek ${ }^{1 *}$

1 Université de Lorraine, CNRS, LPCT, F-54000 Nancy, France

${ }^{*}$ Correspondence: mounir.tarek@univ-lorraine.fr

\section{Supplementary Material}

\begin{tabular}{|c|c|c|c|c|c|c|c|c|}
\hline \multirow{2}{*}{$\begin{array}{l}\text { Charge } \\
\text { reversal } \\
\text { mutants }\end{array}$} & \multirow{2}{*}{ References } & \multirow{2}{*}{ State } & \multicolumn{3}{|c|}{$\begin{array}{c}\text { Number of subunits in } 4 \mathrm{PIP}_{2} \\
\text { systems (over } 4 \text { ) }\end{array}$} & \multicolumn{3}{|c|}{$\begin{array}{c}\text { Number of subunits in } 8 \mathrm{PIP}_{2} \\
\text { systems (over } 4 \text { ) }\end{array}$} \\
\hline & & & AO & IC & RC & AO & IC & RC \\
\hline E160R/R237E & \multirow{5}{*}{$\begin{array}{c}\text { (Wu et al., } \\
\text { 2010a; Zaydman } \\
\text { et al., 2014; Wu } \\
\text { et al., 2010b) }\end{array}$} & $\mathrm{AO}$ & 4 & 0 & 0 & 3 & 0 & 0 \\
\hline E160R/R231E & & IC & 0 & 4 & 2 & 0 & 4 & 0 \\
\hline E170R/R237Q & & IC & 0 & 2 & 1 & 0 & 3 & 0 \\
\hline E160R/R228E & & $\mathrm{RC}$ & 0 & 0 & 4 & 0 & 0 & 4 \\
\hline E170R/R231E & & $\mathrm{RC}$ & 0 & 0 & 1 & 0 & 0 & 4 \\
\hline
\end{tabular}

Table S1: State dependent VSD salt bridges in IK $\mathrm{K}_{\mathrm{S}}$ models

Identification of Kv7.1 state dependent salt-bridges of S4 gating charges, determined through charge reversal mutagenesis, in our IKs models. Each residue name is colored according to the chemical nature of its sidechain. Basic residues in blue, acidic residues in red, and polar residues in green.

\begin{tabular}{|c|c|c|c|c|c|c|c|c|c|c|c|}
\hline \multicolumn{4}{|c|}{ Kv7.1 residue pairs } & \multirow[t]{2}{*}{ State } & \multirow[t]{2}{*}{ Reference } & \multicolumn{3}{|c|}{$\begin{array}{c}\text { Number of subunits } \\
\text { in } 4 \mathrm{PIP}_{2} \text { systems } \\
\text { (over 4) }\end{array}$} & \multicolumn{3}{|c|}{$\begin{array}{c}\text { Number of subunits } \\
\text { in } 8 \mathrm{PIP}_{2} \text { systems } \\
\text { (over } 4)\end{array}$} \\
\hline \multicolumn{2}{|c|}{ Residue 1} & \multicolumn{2}{|c|}{ Residue 2} & & & AO & IC & RC & AO & IC & RC \\
\hline \multirow[b]{2}{*}{ Thr144 } & \multirow[b]{2}{*}{ S1 } & Tyr299 & \multirow[b]{2}{*}{ P-helix } & \multirow[b]{2}{*}{$\mathrm{AO}$} & \multirow{2}{*}{$\begin{array}{c}\text { (Xu et al., } \\
\text { 2013) }\end{array}$} & 4 & 4 & 4 & 4 & 4 & 4 \\
\hline & & $\begin{array}{l}\text { Ser298 } \\
\text { Ala300 }\end{array}$ & & & & $\begin{array}{l}4 \\
4\end{array}$ & 1 & $\begin{array}{l}4 \\
4\end{array}$ & 4 & 0 & $\begin{array}{l}4 \\
3\end{array}$ \\
\hline Phe232 & S4 & Phe279 & S5 & IC & $\begin{array}{l}\text { (Nakajo } \\
\text { and Kubo, } \\
\text { 2014) }\end{array}$ & 4 & 4 & 3 & 4 & 4 & 3 \\
\hline Leu250* & \multirow[b]{2}{*}{ S4-S5 } & \multirow[b]{2}{*}{ Leu353* } & \multirow[b]{2}{*}{ S6 } & \multirow[b]{2}{*}{$\mathrm{RC}$} & \multirow{2}{*}{$\begin{array}{l}\text { (Choveau } \\
\text { et al., } \\
2011 \text { ) }\end{array}$} & 4 & 4 & 4 & 4 & 4 & 4 \\
\hline $\begin{array}{l}\text { Val254* } \\
\text { His258* }\end{array}$ & & & & & & $\begin{array}{l}4 \\
4\end{array}$ & $\begin{array}{l}4 \\
4\end{array}$ & $\begin{array}{l}4 \\
4\end{array}$ & $\begin{array}{l}4 \\
4\end{array}$ & $\begin{array}{l}4 \\
4\end{array}$ & $\begin{array}{l}4 \\
3\end{array}$ \\
\hline
\end{tabular}

Table S2: State-dependent protein-protein interactions involving $\mathrm{K}_{\mathrm{v}} 7.1$ residues.

Identification of the Kv7.1 neighbor residue pairs previously identified through site-directed mutagenesis in our IKs models. The residues involved in intrasubunit interactions are marked with an asterisk. The remaining residue pairs are engaged in intersubunit interactions. Each residue name is colored according to the chemical nature of its sidechain. Apolar residues (aliphatic, aromatic) are written in black, and polar residues in green. 


\begin{tabular}{|c|c|c|c|c|c|c|c|c|c|c|c|}
\hline \multicolumn{4}{|c|}{ Residue Pairs } & \multirow{2}{*}{$\begin{array}{l}\text { Assigned } \\
\text { state }\end{array}$} & \multirow{2}{*}{ Reference } & \multicolumn{3}{|c|}{$\begin{array}{l}\text { Number of subunits in } \\
4 \mathrm{PIP}_{2} \text { systems (over } 4 \text { ) }\end{array}$} & \multicolumn{3}{|c|}{$\begin{array}{l}\text { Number of subunits in } \\
8 \mathrm{PIP}_{2} \text { systems (over } 4 \text { ) }\end{array}$} \\
\hline \multicolumn{2}{|c|}{ KCNE1 region } & \multicolumn{2}{|c|}{ Kv7.1 segment } & & & AO & IC & $\mathrm{RC}$ & AO & IC & $\mathrm{RC}$ \\
\hline Gly40 & $\mathrm{N}_{\text {TERM }}$ & Thr144 & S1 & \multirow{5}{*}{$\mathrm{AO}$} & $\begin{array}{c}\text { (Wang et al., } \\
2011 \text { ) }\end{array}$ & 4 & 0 & 1 & 4 & 0 & 3 \\
\hline Gly40 & $\mathrm{N}_{\text {TERM }}$ & Ile145 & S1 & & $\begin{array}{c}\text { (Xu et al., } \\
\text { 2008) }\end{array}$ & 4 & 2 & 3 & 4 & 0 & 2 \\
\hline Gly40 & $\mathrm{N}_{\text {TERM }}$ & GIn147 & S1 & & $\begin{array}{c}\text { (Wang et al., } \\
\text { 2011) }\end{array}$ & 4 & 0 & 1 & 4 & 0 & 2 \\
\hline Lys41 & $\mathrm{N}_{\text {TERM }}$ & GIn147 & S1 & & $\begin{array}{c}\text { (Wang et al., } \\
\text { 2011) }\end{array}$ & 1 & 1 & 3 & 1 & 0 & 3 \\
\hline Leu42 & $\mathrm{N}_{\text {TERM }}$ & Val324 & S6 & & $\begin{array}{c}\text { (Chung et al., } \\
\text { 2009) }\end{array}$ & 4 & 2 & 1 & 4 & 3 & 1 \\
\hline Lys41 & $\mathrm{N}_{\text {TERM }}$ & Thr144 & S1 & \multirow{6}{*}{$\mathrm{RC}$} & $\begin{array}{c}\text { (Chung et al., } \\
\text { 2009) }\end{array}$ & 2 & 2 & 4 & 3 & 1 & 3 \\
\hline Lys41 & $\mathrm{N}_{\text {TERM }}$ & Ile145 & S1 & & $\begin{array}{c}\text { (Xu et al., } \\
2008)\end{array}$ & 4 & 3 & 4 & 4 & 2 & 3 \\
\hline Lys41 & $\mathrm{N}_{\text {TERM }}$ & Val324 & S6 & & \begin{tabular}{|c|} 
(Chung et al., \\
2009)
\end{tabular} & 0 & 2 & 2 & 0 & 2 & 3 \\
\hline Leu42 & $\mathrm{N}_{\text {TERM }}$ & Ile145 & S1 & & $\begin{array}{c}\text { (Chung et al., } \\
\text { 2009) }\end{array}$ & 4 & 3 & 4 & 4 & 2 & 3 \\
\hline Leu42 & $\mathrm{N}_{\text {TERM }}$ & Gln147 & $\mathrm{S} 1$ & & \begin{tabular}{|c} 
(Wang et al., \\
2011)
\end{tabular} & 0 & 0 & 3 & 0 & 0 & 3 \\
\hline Glu43 & $\mathrm{N}_{\text {TERM }}$ & GIn147 & S1 & & $\begin{array}{c}\text { (Wang et al., } \\
\text { 2011) }\end{array}$ & 2 & 0 & 0 & 1 & 0 & 1 \\
\hline Glu43 & $\mathrm{N}_{\text {TERM }}$ & Trp323 & S6 & \multirow{9}{*}{$\begin{array}{c}\text { Not } \\
\text { specified }\end{array}$} & \begin{tabular}{|c|} 
(Chan et al., \\
2012)
\end{tabular} & 4 & 4 & 4 & 4 & 4 & 4 \\
\hline Leu51 & TMD & Cys331 & S6 & & $\begin{array}{l}\text { (Tapper and } \\
\text { George, } \\
\text { 2001) }\end{array}$ & 3 & 4 & 4 & 4 & 4 & 4 \\
\hline Glu43 & $\mathrm{N}_{\text {TERM }}$ & Val141 & S1 & & $\begin{array}{c}\text { (Chan et al., } \\
\text { 2012) }\end{array}$ & 4 & 3 & 3 & 4 & 2 & 4 \\
\hline Ala44 & $\mathrm{N}_{\text {TERM }}$ & Val141 & $\mathrm{S} 1$ & & $\begin{array}{c}\text { (Chan et al., } \\
\text { 2012) }\end{array}$ & 4 & 3 & 3 & 4 & 3 & 2 \\
\hline Phe54 & TMD & Cys331 & S6 & & $\begin{array}{l}\text { (Tapper and } \\
\text { George, } \\
\text { 2001) }\end{array}$ & 4 & 3 & 4 & 4 & 4 & 2 \\
\hline Leu51 & TMD & Ile328 & S6 & & $\begin{array}{l}\text { (Tapper and } \\
\text { George, } \\
\text { 2001) } \\
\end{array}$ & 0 & 3 & 4 & 0 & 4 & 4 \\
\hline Ser74 & $\mathrm{C}_{\text {TERM }}$ & His363 & $\mathrm{S} 6$ & & $\begin{array}{l}\text { (Lvov et al., } \\
2010)\end{array}$ & 0 & 3 & 2 & 0 & 3 & 2 \\
\hline His73 & $\mathrm{C}_{\text {TERM }}$ & His363 & S6 & & $\begin{array}{l}\text { (Lvov et al., } \\
2010)\end{array}$ & 0 & 1 & 2 & 0 & 1 & 0 \\
\hline Asp76 & $\mathrm{C}_{\text {TERM }}$ & His363 & S6 & & $\begin{array}{l}\text { (Lvov et al., } \\
\text { 2010) }\end{array}$ & 0 & 1 & 4 & 0 & 1 & 2 \\
\hline
\end{tabular}

Table S3: State-dependent protein-protein interactions involving $\mathrm{Kv} 7.1$ and $K C N E 1$ residues.

Identification of the Kv7.1-KCNE1 neighbor residue pairs previously identified through Cysteine cross-linking studies on our IKs models. Each residue is colored according to the chemical nature of its sidechain. Apolar residues (aliphatic, aromatic) are written in black, basic residues in blue, acidic residues in red, and polar residues in green. 


\begin{tabular}{|c|c|c|c|c|c|c|c|c|c|c|c|}
\hline \multirow{3}{*}{ Mutation } & \multirow{3}{*}{ Segment } & \multirow{3}{*}{ Reference } & \multicolumn{9}{|c|}{ Number of subunits in $\mathrm{IK}_{\mathrm{s}}$ models (over 4 ) } \\
\hline & & & \multicolumn{3}{|c|}{$\begin{array}{l}\text { PIP2 intra (4PIP } \\
\text { systems) }\end{array}$} & \multicolumn{3}{|c|}{$\begin{array}{c}\text { PIP2 intra (8PIP }{ }_{2} \\
\text { systems) }\end{array}$} & \multicolumn{3}{|c|}{$\begin{array}{c}\text { PIP2 inter (8PIP } \\
\text { systems) }\end{array}$} \\
\hline & & & AO & IC & $\mathrm{RC}$ & AO & IC & RC & AO & IC & RC \\
\hline Arg190Cys & \multirow{4}{*}{ S2-S3 ${ }_{\text {LOOP }}$} & \multirow{4}{*}{$\begin{array}{l}\text { (Eckey et al., } \\
2014 ; \\
\text { Zaydman et } \\
\text { al., 2013) }\end{array}$} & 4 & 4 & 3 & 4 & 4 & 4 & 0 & 0 & 0 \\
\hline Arg195GIn & & & 4 & 3 & 3 & 4 & 4 & 2 & 0 & 0 & 0 \\
\hline Arg192Cys & & & 2 & 3 & 0 & 3 & 0 & 0 & 0 & 0 & 0 \\
\hline Lys183Cys & & & 3 & 0 & 2 & 2 & 0 & 2 & 0 & 0 & 0 \\
\hline Arg243His & S4 & $\begin{array}{l}\text { (Park et al., } \\
\text { 2005) }\end{array}$ & 0 & 3 & 4 & 0 & 2 & 4 & 0 & 0 & 0 \\
\hline Arg249Cys & S4-S5 $5_{\text {LINKER }}$ & \multirow{2}{*}{$\begin{array}{c}\text { (Eckey et al., } \\
2014 ; \\
\text { Zaydman et } \\
\text { al., 2013) }\end{array}$} & 3 & 4 & 4 & 2 & 4 & 4 & 1 & 0 & 0 \\
\hline Lys354Cys & \multirow{4}{*}{ S6 } & & 3 & 3 & 1 & 2 & 2 & 0 & 0 & 0 & 0 \\
\hline Lys358Cys & & \multirow{3}{*}{$\begin{array}{l}\text { (Thomas et } \\
\text { al., 2011; } \\
\text { Zaydman et } \\
\text { al., 2013) }\end{array}$} & 1 & 2 & 1 & 1 & 0 & 0 & 3 & 2 & 1 \\
\hline Lys362Cys & & & 0 & 0 & 0 & 0 & 0 & 0 & 4 & 4 & 3 \\
\hline Arg366Ala & & & 1 & 0 & 1 & 0 & 0 & 0 & 4 & 4 & 3 \\
\hline Arg67Gln & \multirow{3}{*}{$\begin{array}{c}\text { KCNE1 } \\
\text { CTER } \\
M\end{array}$} & \multirow{3}{*}{$\begin{array}{l}\text { (Li et al., } \\
\text { 2011) }\end{array}$} & 1 & 0 & 0 & 1 & 0 & 0 & 0 & 4 & 4 \\
\hline Lys69Cys & & & 0 & 4 & 2 & 0 & 4 & 2 & 4 & 0 & 0 \\
\hline Lys70Cys & & & 0 & 0 & 0 & 0 & 1 & 0 & 0 & 1 & 3 \\
\hline
\end{tabular}

Table S4: State-dependent protein-lipid interactions between $\mathrm{Kv}_{\mathrm{v}}$.1/KCNE1 basic residues and PIP $_{2}$. Identification of the basic residues from Kv7.1 and KCNE1 subunits which have been previously shown to interact with $\mathrm{PIP}_{2}$ through neutralizing point mutations. In the first column, residue names are colored according to its sidechain chemical nature. Apolar residues are colored in black, basic residues in blue, and polar residues in green.

Resting state

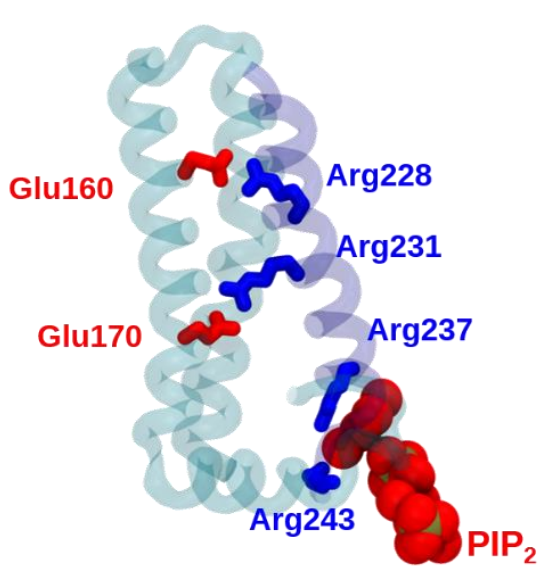

Intermediate state

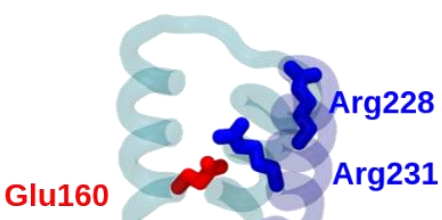

Glu170

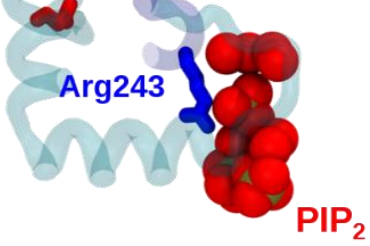

Activated state

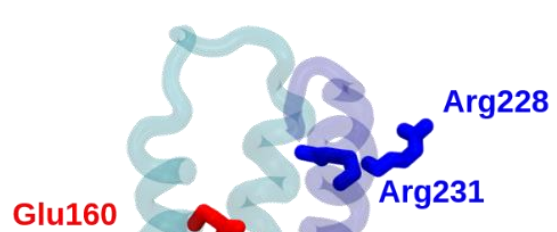

Arg237

Glu170

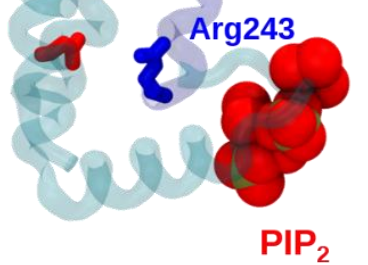

Figure S1: State-dependent ionic interactions in the VSD of Kv7.1 subunits

Mapping of S4 gating charges (in blue sticks) along with their VSD countercharges (in red sticks) and $\mathrm{PIP}_{2}$ intra phosphate groups (in red spheres) in stable state models of IKs and Kv7.1 channels. VSD segments are represented in transparent cyan ribbons, except S4 (in transparent blue ribbons) and S3 segment, hidden for clarity. 

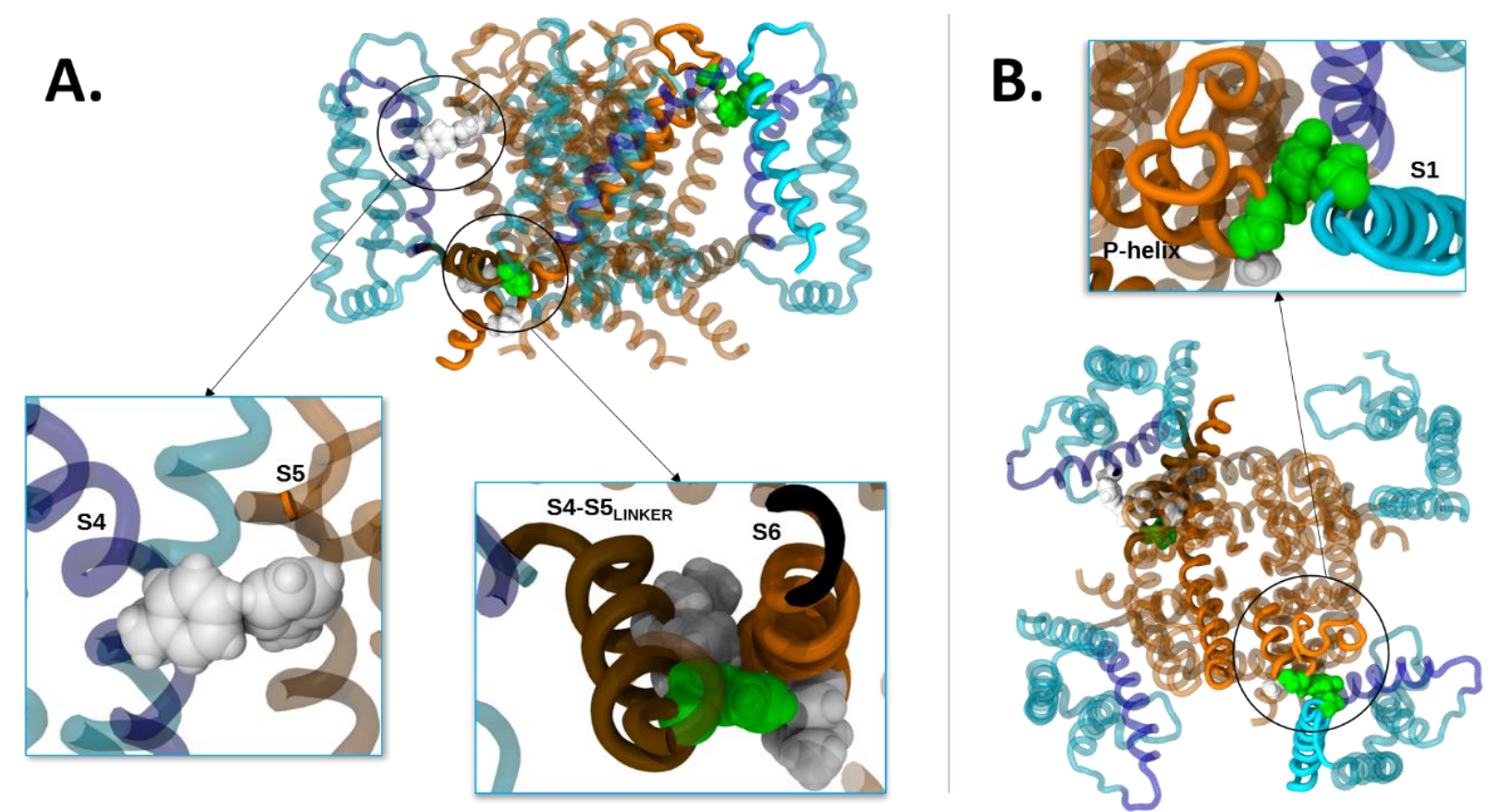

Figure S2: State-dependent $\mathrm{K}_{\mathrm{v}} 7.1$ protein-protein interactions in IK $\mathrm{K}$ models.

Structural mapping of state-dependent protein-protein interactions in our IKs models in A. Side view and B. Top view. KCNE1 segments are hidden for clarity. Kv7.1 segments are represented in transparent ribbons, except those which carry the residue pairs of interest, which are in solid colors. Neighbor residue pairs are circled in black, and link to a zoomed view. For transmembrane segments, VSD ones are colored in cyan, S4 in blue, S4S5LINKER in brown and PD segments in orange. Residues of interest are colored according to the chemical nature of their sidechains: apolar residues are colored in white, and polar residues are in green 


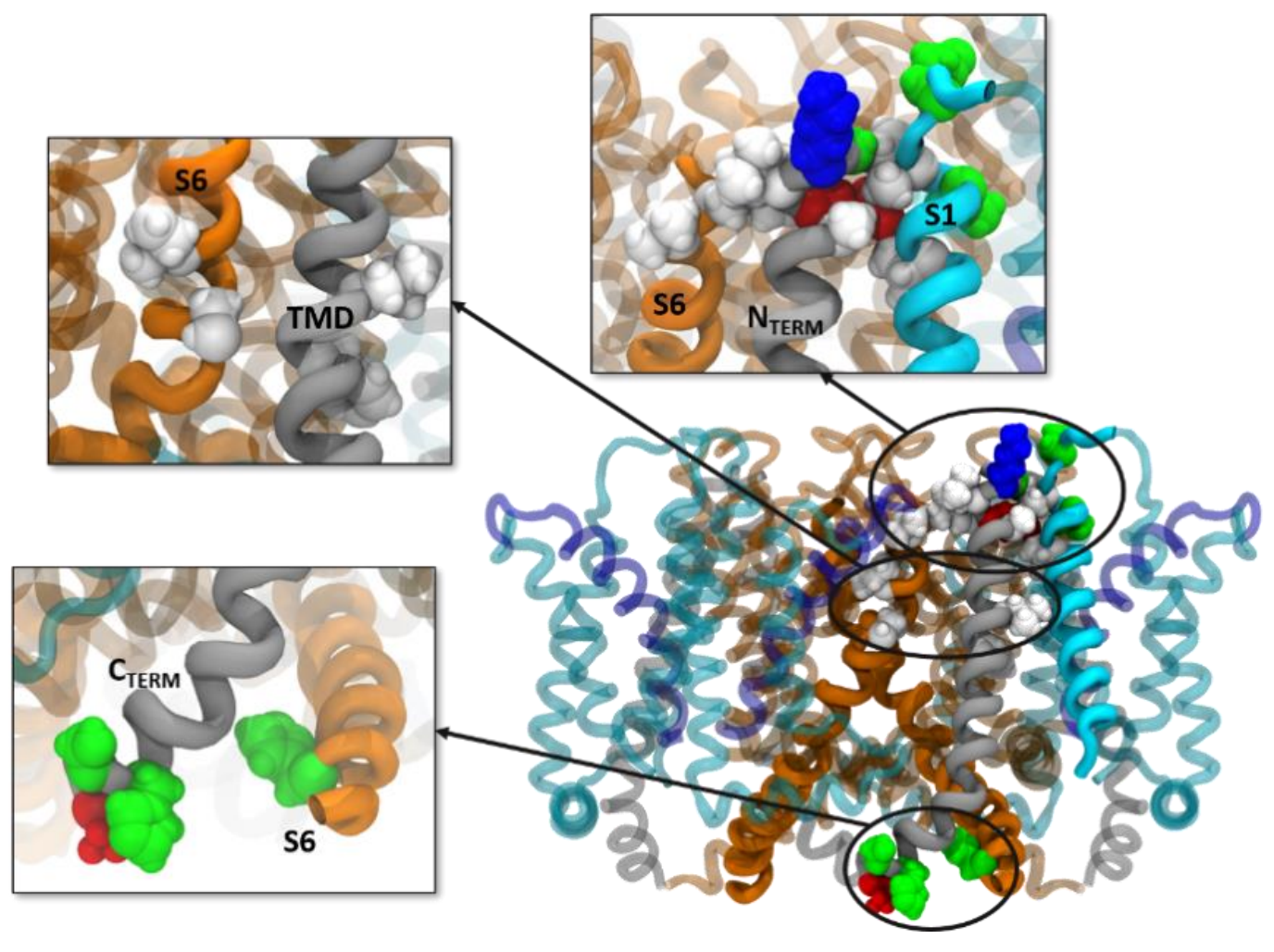

Figure S3: State-dependent KCNE1-Kv7.1 interactions in IK models

Structural mapping of state-dependent protein-protein interactions in IKs models. KCNE1 segments are represented in grey ribbons. Kv7.1 segments are represented in transparent ribbons, except those containing the residue pairs of interest, which are in solid colors. Neighbor residue pairs are circled in black, and for which a zoomed view is provided. For transmembrane segments, the color code used is the same as those of Figure S2. Residues of interest are colored according to the chemical nature of their sidechains: apolar residues are colored in white, acidic residues in red, basic residues in blue, and polar residues in green. 

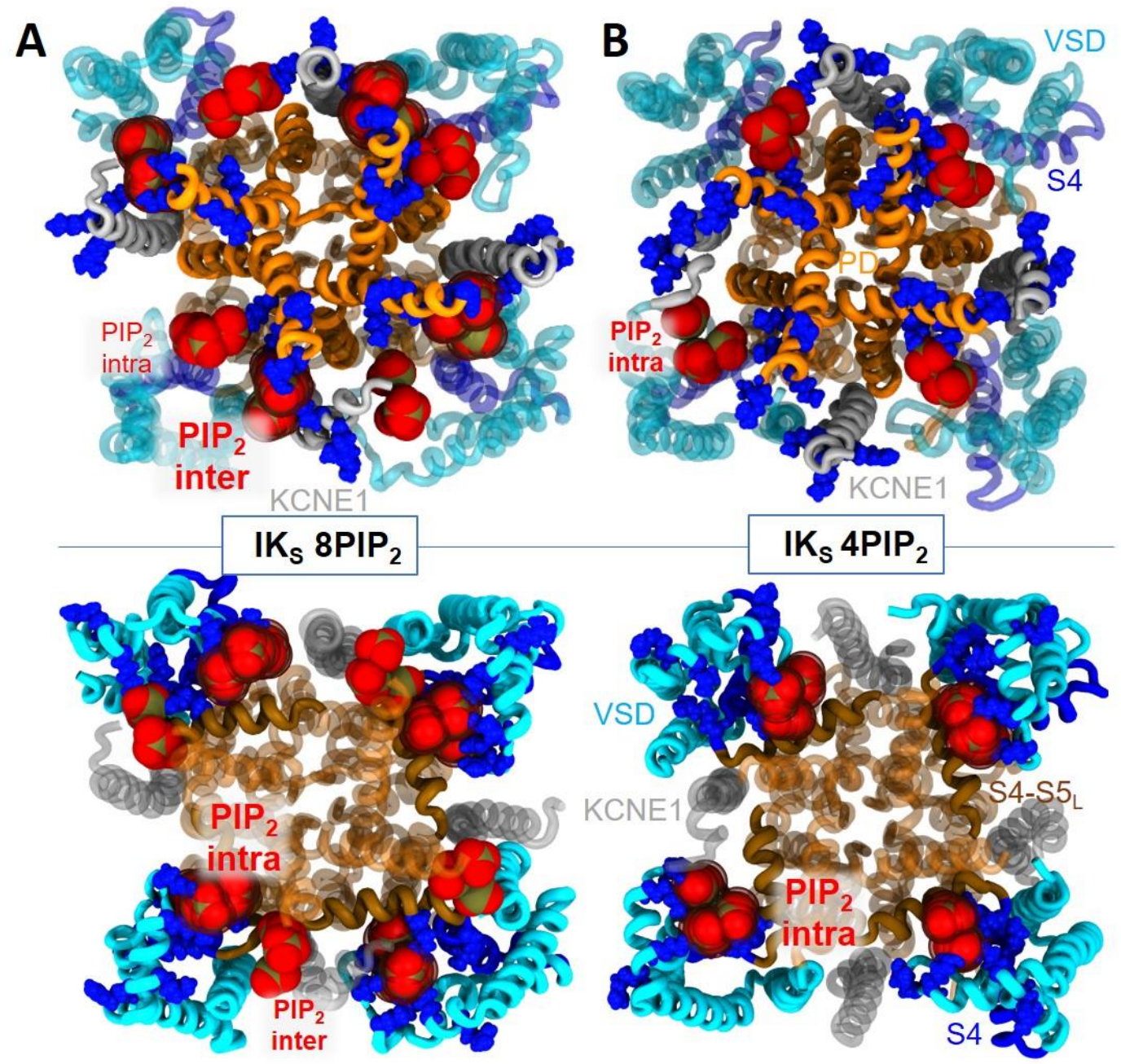

Figure S4: Structural mapping of the salt-bridges between $\mathrm{IK}_{\mathrm{S}}$ complex residues and $\mathrm{PIP}_{2}$.

Cytoplasmic view of the transmembrane subunits (in ribbons) of the AO models of IKs complex. VSD segments are colored in cyan, S4 segments in blue, PD segments in orange and KCNE1 subunits in gray. In each panel, the basic residues of IKs complex are depicted in blue spheres, while the charge moieties of $\mathrm{PIP}_{2}$ inter and $\mathrm{PIP}_{2}$ intra are depicted in bright red and dark red spheres, respectively. A. In 8PIP 2 systems, the upper panel shows the cytoplasmic regions of KCNE1 and S6 (in solid colors) which specifically interact with $\mathrm{PIP}_{2}$ inter. The lower panel shows the cytoplasmic regions of the VSD including S2-S3LOOP, S4 and S4-S5LINKER (in solid colors) that interact with $\mathrm{PIP}_{2}$ intra. B. In 4PIP 2 systems, the upper panel depicts the relative position of the basic residues of KCNE1 (in solid colors) with respect to $\mathrm{PIP}_{2}$ intra that interacts with the cytoplasmic region of S6, as $\mathrm{PIP}_{2}$ inter lipids are absent. The lower panel shows the interactions between the basic residues from S2-S3Loop, S4 and S4-S5 LINKER (in solid colors) that interact with $\mathrm{PIP}_{2}$ intra (in circled spheres). 